Check for updates

Cite this: Phys. Chem. Chem. Phys., 2021, 23, 7988

Received 16th November 2020

Accepted 6th January 2021

DOI: 10.1039/d0cp05943a

rsc.li/pccp

\section{Syngas molecules as probes for defects in 2D hexagonal boron nitride: their adsorption and vibrations $\dagger$}

\author{
Tao Jiang, (D) a Duy Le, (D) ${ }^{\text {ab }}$ Takat B. Rawal (D) † $^{\mathrm{a}}$ and Talat S. Rahman (D) *ab
}

Single-layer, defect-laden hexagonal boron nitride $(\mathrm{dh}-\mathrm{BN})$ is attracting a great deal of attention for its diverse applications: catalysis on the one hand, and single photon emission on the other. As possible probes for identifying some common defects in single-layer $\mathrm{h}-\mathrm{BN}$, we present results of ab initio calculations for the adsorption and vibrational characteristics of syngas molecules $\left(\mathrm{H}_{2}, \mathrm{CO}, \mathrm{CO}_{2}\right)$ on dh-BN containing one of four types of defects: nitrogen vacancy $\left(V_{N}\right)$, boron vacancy $\left(V_{B}\right)$, Stone-Wales defect $(S W)$, and nitrogen substituted by boron $\left(B_{N}\right)$. Through a comparative examination of adsorption features, charge transfer, electronic structure, and vibrational spectrum, we obtain a deep understanding of the interaction of these molecules with $d h-\mathrm{BN}$ and the role of the defect states. We find that while $\mathrm{CO}, \mathrm{CO}_{2}$ and atomic hydrogen chemisorb, molecular $\mathrm{H}_{2}$ physisorbs on $d h-\mathrm{BN}$ with the four considered defect types. $\mathrm{V}_{\mathrm{N}}$ and $\mathrm{V}_{\mathrm{B}}$ show strong affinity for $\mathrm{CO}$ and $\mathrm{CO}_{2}$ since the defect states induced by them lie close to the Fermi level. SW does not favor adsorption of these small molecules, as the process for each is endothermic. In the case of $\mathrm{B}_{\mathrm{N}}, \mathrm{CO}$ adsorbs strongly but $\mathrm{CO}_{2}$ only weakly. Vibrational frequencies of notable modes localized at the adsorbed molecules are analyzed and suggested as measures for identification of the defect type. Through a simple comparison of adsorption characteristics of the molecules on these defects, we propose $d h-\mathrm{BN}$ with $\mathrm{V}_{\mathrm{N}}$ to be a good catalyst candidate for $\mathrm{CO}_{2}$ hydrogenation.

\section{Introduction}

Similar to other two dimensional (2D) materials, single-layer hexagonal boron nitride $(h$-BN) has received considerable attention in the past few years owing to its novel properties that make it amenable for various applications. ${ }^{1}$ Structurally analogous to graphene, single-layer $h$-BN has B and $\mathrm{N}$ atoms arranged in a hexagonal lattice and bound together by strong covalent bonds. It is a wide bandgap material with high chemical stability ${ }^{2}$ and ideally suited as a reliable support for other $2 \mathrm{D}$ materials. ${ }^{3}$ Its unique properties have also suggested its potential usage in optoelectronics ${ }^{4}$ and photo-catalysis. ${ }^{5}$ Even more remarkable have been the recent findings of its suitability as a thermal catalyst ${ }^{1,6,7}$ and as a single photon emitter, ${ }^{8-10}$ pointing to the important role of defects. This is to be expected as pristine $h$-BN is chemically inert because of

\footnotetext{
${ }^{a}$ Department of Physics, University of Central Florida, Orlando, FL, 32816, USA. E-mail: talat.rahman@ucf.edu

${ }^{b}$ Renewable Energy and Chemical Transformations Cluster, University of Central Florida, Orlando, FL, 32816, USA

$\dagger$ Electronic supplementary information (ESI) available. See DOI: 10.1039/ d0cp05943a

\# Present address: Department of Physics, University of Houston, Houston, TX 77204, USA.
}

the lack of dangling bonds. ${ }^{11}$ It therefore calls for prudent ways to activate the basal plane of $h$-BN. Taking cues from prior studies on $\operatorname{MoS}_{2},{ }^{12-17}$ proposals have been made to activate single-layer $h$-BN by the creation of defects, ${ }^{18,19}$ deposition of metal atoms or molecules, ${ }^{20-23}$ creation of grain-boundaries, ${ }^{24}$ and application of strain to the defective surface. ${ }^{25}$ Quite akin to defect-laden single-layer $\mathrm{MoS}_{2}$ (with sulfur vacancies), which calculations predict to be a possible catalyst for hydrogenation of syngas to alcohol, ${ }^{12}$ single-layer $h$-BN can be chemically activated for similar reactions, in the presence of point or line defects. Indeed, in the above mentioned experimental and theoretical study ${ }^{6}$ showing propane hydrogenation, the activity was traced to the presence of defects. While nitrogen vacancy has been proposed as the most active defect, interestingly for both catalytic ${ }^{6}$ and single-photon emissive ${ }^{8}$ properties, the role of other types of defects cannot be ruled out. Defects are, however, not easy to characterize experimentally, notwithstanding the nanometer resolution scanning tunnelling microscopy (STM) studies by Feng et al. ${ }^{9}$ Small molecules such as $\mathrm{H}_{2}, \mathrm{CO}, \mathrm{CO}_{2}$, the syngas trio, could, however, serve as a probe of defects through their adsorption and vibrational characteristics on defect-laden hexagonal boron nitride $(d h$-BN).

Beyond serving as probes of defects, an in-depth understanding of the relative propensity of common defect types in facilitating 
adsorption of syngas molecules on $d h-\mathrm{BN}$ is the first step in rational designing of $d h$-BN for specific hydrogenation reactions. The topic is of broad interest since $h$-BN is readily available and thermally stable. With appropriate defects it could serve as a metal-free catalyst for hydrogenation of $\mathrm{CO} / \mathrm{CO}_{2}$ even at temperatures higher than ambient. To probe the catalytic propensity of $h$-BN toward such hydrogenation, it is thus essential that we gain fundamental insights into the interactions between $h$-BN and syngas, as presented in this work. It should be noted that adsorption of $\mathrm{H}_{2}, \mathrm{CO}$, and $\mathrm{CO}_{2}$ (syngas) on other catalysts leading to promising reaction pathway of their conversion into useful (value-added) chemical products, i.e., simple to higher alcohols, formaldehyde, formic acid, and higher hydrocarbons has been the subject of several studies. ${ }^{26-28}$ Furthermore, thermodynamic and kinetic favorability of adsorption of syngas molecules on the catalyst surface are critical for efficiently and selectively promoting hydrogenations of $\mathrm{CO}$ and $\mathrm{CO}_{2}{ }^{29}$ Additionally, catalyst material should be thermally stable, chemically active and selective for the desired reaction products. Historically, metal-based catalysts have been widely investigated for syngas hydrogenation reactions, as they typically demonstrate good catalytic activity and product selectivity. ${ }^{26-28}$ The optimal catalyst material is, however, often an expensive, non-abundant metal and/ or can be easily oxidized and deactivated. Copper-based catalysts ${ }^{28}$ are, of course, not expensive but they have poor thermal stability, and hence not suitable for high-temperature reactions without a thermal stabilizer. Cerium-based catalysts ${ }^{30}$ have also shown good activity and selectivity for $\mathrm{CO}_{2}$ hydrogenation, but are easily deactivated by a small coverage of deposited carbon. There is thus the need to develop more cost-effective catalyst than presently available.

With the above in mind, we present here results of ab initio calculations, based on density functional theory (DFT) of the adsorption and vibrational characteristics of syngas on $d h$-BN with one of four possible types of defects - nitrogen vacancy $\left(\mathrm{V}_{\mathrm{N}}\right)$, boron vacancy $\left(\mathrm{V}_{\mathrm{B}}\right)$, Stone-Wales (SW), and nitrogen substituted by boron $\left(B_{N}\right)$ - which have been shown to have affinity towards the selected adsorbates. ${ }^{6}$ We examine favorable adsorption sites and orientation of these molecules on $d h$-BN and analyze the unique local atomic environment and electronic structure of $d h$-BN as modified by the adsorbed molecules $\left(\mathrm{H}_{2}, \mathrm{CO}\right.$ and $\left.\mathrm{CO}_{2}\right)$. Quite importantly, we calculate and analyze vibrational frequencies of modes localized at the adsorbed molecules and nearby defect sites to identify notable modes as possible signatures of the adsorption, and thereby the defect type. Based on the DFT-calculated thermodynamics for syngasadsorption, we suggest that the $h$-BN with nitrogen vacancy may serve as a potential metal-free catalyst material for hydrogenation of $\mathrm{CO}_{2}$, fully recognizing that the results presented here are only a first step towards a comprehensive understanding of catalyst reactivity and selectivity.

In the next section, we introduce details of our computational methodology. We next present results and discussion, starting with the geometrical and electronic structure of the four types of point defects in $h$-BN considered in this work, followed by the adsorption and vibrational analysis of $\mathrm{H}_{2}, \mathrm{CO}$, and $\mathrm{CO}_{2}$ on the defects and discussion of implications of our results towards the catalytic activity of $h$-BN. Finally, we provide main conclusions of this work.

\section{Computational details}

All calculations are performed by using spin-polarized DFT, implemented in the Quantum Espresso Code, ${ }^{31}$ employing plane-wave basis set and the projected-augmented-wave (PAW) method $^{32,33}$ for interaction between electrons and nuclei. We use the vdW-DF2 functional ${ }^{34}$ for exchange-correlation of electrons as it is found to produce excellent lattice constant of $h$-BN. We use the plane wave basis set with kinetic energy cut-off of 45 Ry and charge density with $180 \mathrm{Ry}$. We construct a $(6 \times 6)$ supercell of single layer hexagonal boron nitride $(h$-BN) with periodic boundary conditions along in-plane directions and a vacuum of $20 \AA$ to avoid the spurious interaction between periodical images. To model a defect-laden $h$-BN system $(d h$-BN), we remove either a boron or a nitrogen atom or substitute a nitrogen atom by boron or rotate a B-N bond by $90^{\circ}$. The lateral dimensions of the resulting $d h$-BN are optimized so that the system is stress free. The relaxed structures of our model systems of $h$-BN with the four defects are shown in Fig. 1. To investigate the adsorption characteristics of $\mathrm{H}_{2}, \mathrm{CO}$ and $\mathrm{CO}_{2}$ we begin by placing a molecule at the defect site and at the neighboring atoms near the defect sites and perform ionic relaxations to obtain the lowest-energy adsorption configuration. All systems are relaxed until their energy converges with a threshold of $10^{-6} \mathrm{eV}$ and the residual force on each atom reaches below $0.005 \mathrm{eV} \AA^{-1}$. For calculations of relaxed geometry, we sample the Brillouin zone with one $k$-point (zone center: $\Gamma$ point) and for electronic density of states, we use a $12 \times 12 \times 1 k$-point
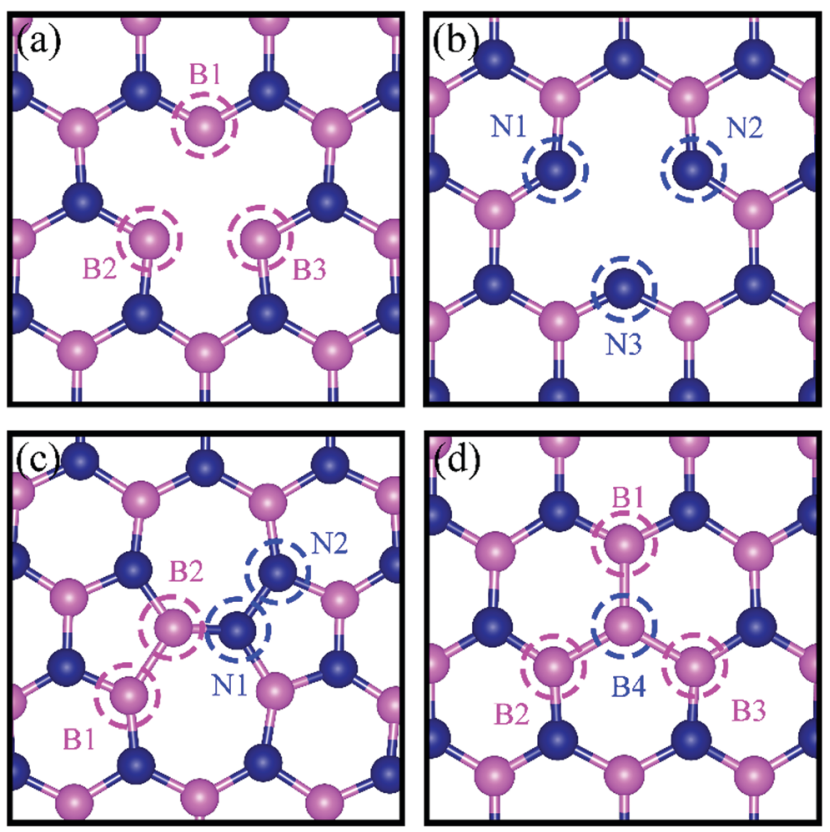

Fig. 1 Schematic representation of $h$-BN model systems with defects: (a) $V_{N}$, (b) $V_{B}$, (c) $S W$, and (d) $B_{N}$. Blue and magenta balls represent $N$ and $B$ atoms, respectively. 
mesh. As shown in the ESI, $\dagger$ usage of $\Gamma$-point sampling produces system total energy within $7 \mathrm{meV}$ of that obtained with larger $k$-point sampling, thereby justifying our usage of $\Gamma$ point sampling.

The adsorption energy is defined as $E_{\mathrm{ads}}=E_{\text {Molecule } / \mathrm{d} h \text {-BN }}-$ $\left(E_{\text {Molecule }}+E_{d h \text {-BN }}\right)$, where $E_{\text {Molecule } / d h-\mathrm{BN}}, E_{\text {Molecule }}, E_{d h \text {-BN }}$ are the total energy of the molecule on $d h$-BN, isolated molecule, and $d h$-BN, respectively. We calculate charge density difference upon adsorption of molecules on $d h$-BN using: $\Delta \rho=\rho_{\text {Molecule } / d h \text {-BN }}{ }^{-}$ $\left(\rho_{\text {Molecule }}+\rho_{d h \text {-BN }}\right)$, where $\rho_{\text {molecule } / d h-\mathrm{BN}}, \rho_{d h-\mathrm{BN}}$, and $\rho_{\text {Molecule }}$ are charge density of the molecule on $d h$-BN, $d h$-BN itself, and the isolated molecule, respectively. The charge on each atom is calculated using Bader analysis ${ }^{35,36}$ which allows estimation of the charge transfer between the molecule and $d h$-BN. Vibration frequencies were calculated using the finite-difference method as implemented in the Phonopy package, ${ }^{37}$ in which we use a displacement of $0.01 \AA$ to maintain validity of the harmonic approximation and avoid numerical errors that would otherwise introduce chaotic behavior in the result. Zero-point energy (ZPE) correction for the total energy of each system is calculated as $\sum_{i=1}^{N} \hbar \nu_{i} / 2$, where $\hbar$ is the Planck constant, $\nu_{i}$ is the frequency of vibrational mode $i(i=1 \ldots N$, where $N$ is the total number of vibrational modes of the system).

\section{Results and discussions}

\section{Geometrical and Electronic Structure of $d h$-BN}

We present in Fig. 1 the geometry of the considered four types of defects in single-layer $h$-BN, $\mathrm{V}_{\mathrm{N}}, \mathrm{V}_{\mathrm{B}}, \mathrm{SW}$, and $\mathrm{B}_{\mathrm{N}}$. To explore their electronic structure, we show the spin dependent density of states of each defect in Fig. 2.

\section{Nitrogen vacancy $\left(V_{N}\right)$}

The presence of a nitrogen vacancy on $h$-BN leads to a local geometric change (Fig. 1a) as reflected by changes in bond lengths and bond angles. The shortest $\mathrm{B}$ and $\mathrm{N}$ bond length $\left(d_{(\mathrm{B} 2-\mathrm{N})}\right)$ is $1.492 \AA$, to be compared to $1.455 \AA$ of pristine $h$-BN, whereas the angle between $\mathrm{N}, \mathrm{B}$ and $\mathrm{N}$ atoms $\left(\theta_{(\mathrm{N}-\mathrm{B} 2-\mathrm{N})}\right)$ is $113.6^{\circ}$ as opposed to $120^{\circ}$ in pristine $h$-BN. Also, the separation between $\mathrm{N}$ atoms, $d_{(\mathrm{N}-\mathrm{N})}$, is changed from 2.485 to $2.471 \AA$ (0.5\% change).

From spin-polarized calculations of electronic density of states in Fig. 2a, we find that $d h$ - $\mathrm{BN}\left(\mathrm{V}_{\mathrm{N}}\right)$ is a spin-polarized system - the effect arising from the unpaired electrons produced by the $\mathrm{N}$ vacancy. Of critical importance are the two midgap states, one is spin-up-the highest occupied molecular orbital (HOMO)-and the other is spin-down-the lowest unoccupied molecular orbital (LUMO). These states are separated by $0.98 \mathrm{eV}$. The HOMO and LUMO wave functions (inserts in Fig. 2a) adapt the same symmetry and are $\pi$-type dominated by $\mathrm{p}_{\mathrm{z}}$ orbitals of B1, B2 and B3 atoms (see Fig. 1a).

\section{Boron vacancy $\left(\mathbf{V}_{\mathbf{B}}\right)$}

The existence of a boron vacancy on single-layer $h$-BN leads to the following geometrical change at the defect (Fig. 1b): as compared to those in pristine $h$ - $\mathrm{BN}$, the bond between $\mathrm{N}$ and $\mathrm{B}$
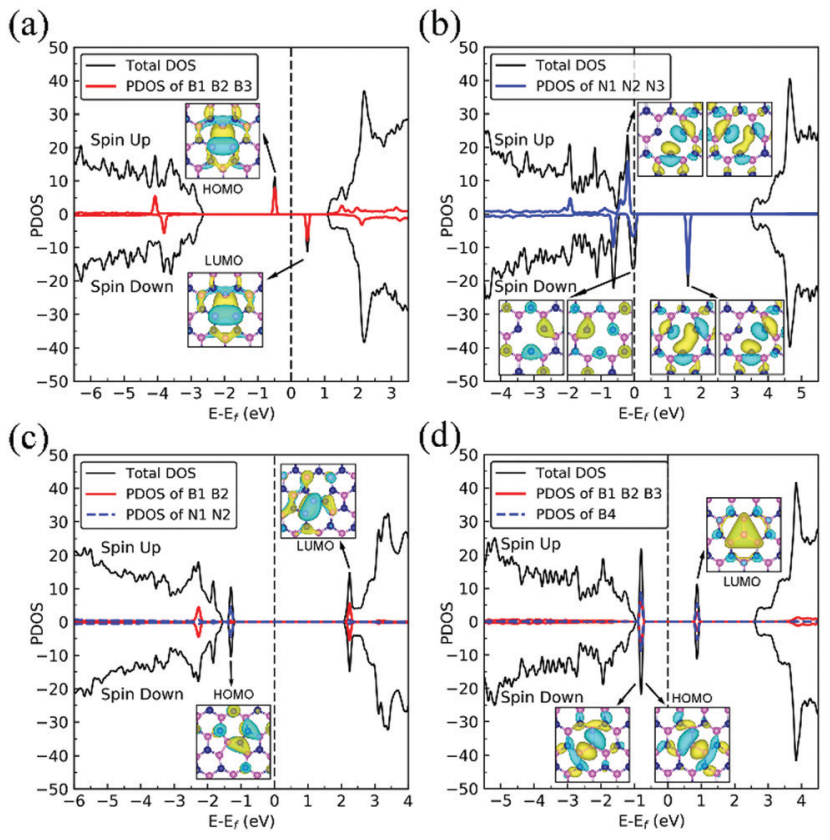

(d)

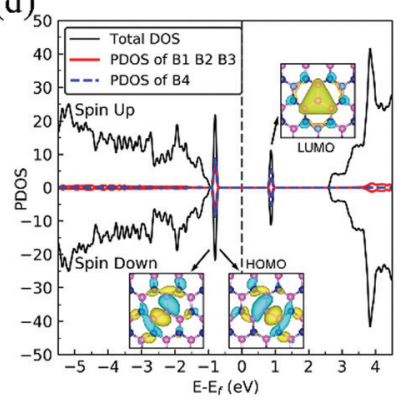

Fig. 2 Spin-polarized projected density of states (PDOS) of $d h$-BN with defects: (a) $V_{N}$, (b) $V_{B}$, (c) $S W$, and (d) $B_{N}$. The inset displays the wave function of the defect states with isosurface value of $0.001 \mid \mathrm{el} \AA^{-1}$.

atoms $\left(d_{(\mathrm{B}-\mathrm{N} 1)}\right)$ shortens from 1.455 to $1.410 \AA$ (3.1\% change); the angle between $\mathrm{B}, \mathrm{N}$ and $\mathrm{B}$ atoms $\left(\theta_{(\mathrm{B}-\mathrm{N} 1-\mathrm{B})}\right)$ enlarges from $120^{\circ}$ to $126.6^{\circ}$ (5.5\% change); and the distance between $\mathrm{N}$ atoms, $d_{(\mathrm{N}-\mathrm{N})}$, elongates from 2.520 to $2.662 \AA$ ( $5.6 \%$ change). The $d h$-BN with $\mathrm{V}_{\mathrm{B}}$ also possesses spin-polarized electronic DOS, as indicated by the calculated spin-up and -down DOS shown in Fig. 2b. The presence of B vacancy leads naturally to undercoordinated nearest-neighboring $\mathrm{N}$ atoms, N1, N2, and N3 (Fig. 1b). The PDOS (blue curve Fig. 2b) show the mid-gap state (above the Fermi level) to be composed mainly of contributions from the N1/N2/N3 atoms in the spin down channel, On the other hand, the half-occupied state at the Fermi level, has partial contributions from N1/N2/N3, and also contributions from other surrounding $\mathrm{N}$ atoms (see inset). These results are in qualitative agreement with previous studies. $^{38-40}$

\section{Stone-Wales (SW) defect}

The Stone-Wales (SW) defect is created by rotation of a B-N bond by $90^{\circ}$ around its midpoint, as shown in Fig. 1c. The bond lengths of B1-B2, B2-N1, N1-N2 are found to be 1.686, 1.375 and $1.478 \AA$, respectively. The PDOS of $d h$-BN (SW), shown in Fig. 2c, indicates that occupied and unoccupied defect states are not spin-polarized. The unoccupied defect states (LUMO) above the Fermi level are mainly contributed by the B1 and B2 atoms with a small contribution from the atoms surround B1 and B2 while the occupied defect states (HOMO) below the Fermi level have main contributions from N1 and N2 atoms. The LUMO states are $\sigma$-type dominated by $\mathrm{p}_{x}$ and $\mathrm{p}_{y}$ orbitals from the B-B bond, while the HOMO states are $\pi$-type, in agreement with ref. 41 . 


\section{B substitution for $\mathbf{N}\left(\mathbf{B}_{\mathbf{N}}\right)$}

$\mathrm{B}_{\mathrm{N}}$ defects are formed by the substitution of a B atom for $\mathrm{N}$ at the lattice of pristine $h$-BN as shown in Fig. $1 \mathrm{~d}$. The B-B and $\mathrm{N}-\mathrm{B}$ bond lengths are found to be 1.627 and $1.452 \AA$, respectively. The PDOS plot, Fig. $2 \mathrm{~d}$, for $d h$-BN with $\mathrm{B}_{\mathrm{N}}$ displays a large bandgap with non-spin-polarized defect-related gap states. Since boron has two fewer valence electrons than nitrogen, dangling bonds are formed. In the defect levels below and above the Fermi level, the atom B4 makes larger contribution than the sum of the contributions from atoms B1, B2, and B3. Note that $\mathrm{B} 4$ and their surrounding boron atoms in single layer $d h$ - $\mathrm{BN}\left(\mathrm{B}_{\mathrm{N}}\right)$ resemble the geometry in the boron nitride nanotube with $\mathrm{B}_{\mathrm{N}}$ defect and display similar electronic structure to those found by $\mathrm{H}$. Choi et $a l^{42}$ We also find that the $\mathrm{B}_{\mathrm{N}}$ defect introduces unoccupied defect states (LUMO) in the middle of the energy gap and occupied defect states (HOMO) near the valence band edge. These are $\sigma$-type orbitals with similar geometric structure in comparison with those in the nanotube with $\mathrm{B}_{\mathrm{N}} \cdot{ }^{42}$

\section{Adsorption of small molecules on $d h-B N$}

\section{Hydrogen molecule on $\boldsymbol{d h}$-BN}

Fig. 3 shows the optimized geometries of $\mathrm{H}_{2}$ on $d h-\mathrm{BN}$ with $\mathrm{V}_{\mathrm{N}}$, $\mathrm{V}_{\mathrm{B}}$, SW, and $\mathrm{B}_{\mathrm{N}}$. We find that the $\mathrm{H}_{2}$ molecule only physisorbs on $d h$-BN with adsorption energy of $-0.07,-0.05,-0.08$ and $-0.08 \mathrm{eV}$, at $\mathrm{V}_{\mathrm{N}}, \mathrm{V}_{\mathrm{B}}, \mathrm{SW}$ and $\mathrm{B}_{\mathrm{N}}$, respectively. The $\mathrm{H}-\mathrm{H}$ bond length on all four defects is $0.737 \AA$, which is almost identical to that in the gas phase $(0.736 \AA)$. The $\mathrm{H}-\mathrm{B}$ bond lengths, $d_{(\mathrm{H}-\mathrm{B})}$, are $3.359,3.267$, and $3.357 \AA$, for $\mathrm{H}_{2}$ on $\mathrm{V}_{\mathrm{N}}, \mathrm{SW}$ and $\mathrm{B}_{\mathrm{N}}$, respectively. Similarly, the $\mathrm{H}-\mathrm{N}$ bond length, $d_{(\mathrm{H}-\mathrm{N})}$, for $\mathrm{H}_{2}$ on $\mathrm{V}_{\mathrm{B}}$ is $3.285 \AA$. Electron transfer from $d h$-BN to $\mathrm{H}_{2}$ is found to be negligible $\left(0.005,0.004,0.002\right.$, and 0.004 electrons, on $V_{N}, V_{B}$, $\mathrm{SW}$, and $\mathrm{B}_{\mathrm{N}}$, respectively). Such small electron transfer reflects the fact that the $\mathrm{H}_{2}$ molecule is physisorbed on $d h$-BN, consistent with almost-unchanged $\mathrm{H}-\mathrm{H}$ bond lengths.

\section{Adsorption of two atomic hydrogens on $\boldsymbol{d h}$-BN}

Since $\mathrm{H}_{2}$ does not chemisorb in molecular form on $d h$-BN with $\mathrm{V}_{\mathrm{N}}, \mathrm{V}_{\mathrm{B}}$, SW and $\mathrm{B}_{\mathrm{N}}$, we turn to investigate the adsorption of the dissociated molecule in the form of two atomic hydrogens on $d h$-BN. Binding geometries of two atomic hydrogens $(2 \mathrm{H})$ on

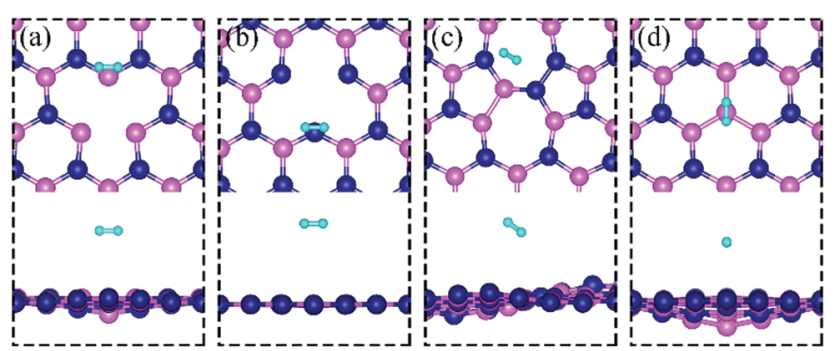

Fig. 3 Optimized structures of $\mathrm{H}_{2}$ adsorbed at favored sites of $h$-BN with (a) $V_{N}$, (b) $V_{B}$, (c) SW, and (d) $B_{N}$. The magenta, blue, white balls represent the $\mathrm{B}, \mathrm{N}$ and $\mathrm{H}$ atoms, respectively. The top and bottom panel shows the top and side views, respectively. the four considered types of $d h$-BN are shown in Fig. 4. The geometries of the most stable adsorption sites are similar to those in previous work by Nash et al. ${ }^{6}$ The calculated energetic and geometric parameters, including the adsorption energy, $\mathrm{H}-\mathrm{H}$ distance, and the distance of formed $\mathrm{B}(\mathrm{N})-\mathrm{H}$ bond, are summarized in Table 1.

On $d h$-BN with $\mathrm{V}_{\mathrm{N}}$, two hydrogen atoms prefer to bind to $\mathrm{B}$ atoms near $\mathrm{N}$ vacancy such that they are on the opposite side of the $d h$-BN sheet, with respect to each other, and the difference in their z coordinates is $2.938 \AA$ (Fig. 4a). The adsorption energy of $2 \mathrm{H}$ is found to be $-1.61 \mathrm{eV}$. On $d h$-BN with $\mathrm{V}_{\mathrm{B}}$, shown in Fig. $4 \mathrm{~b}, 2 \mathrm{H}$ prefer to attach to $\mathrm{N}$ atoms near the $\mathrm{B}$ vacancy in such a way that again the two $\mathrm{H}$ atoms are on the opposite side of the sheet but now with a difference in their z-position of $1.550 \AA$. The adsorption energy of $2 \mathrm{H}$ on $d h$-BN with $\mathrm{V}_{\mathrm{B}}$ is $-4.74 \mathrm{eV}$, an extremely exothermic adsorption. For $2 \mathrm{H}$ adsorption on $d h$-BN with $\mathrm{SW}$, the $\mathrm{H}$ atoms prefer to stay on the same side of the sheet, as shown in Fig. 4c, in which one hydrogen attaches to B3 while the other attaches to N1. We find that the adsorption of $2 \mathrm{H}$ atoms on SW breaks the bond of B3-N1, with the distance $d_{(\mathrm{B} 3-\mathrm{N} 1)}$ of $2.632 \AA$, largely increased from $1.492 \AA$ in the absence of the $\mathrm{H}$ atoms. This adsorption is endothermic with energy of $+1.16 \mathrm{eV}$, the positive sign signifying that it is not favored, mainly because there is no undercoordinated site at SW. For the case of $2 \mathrm{H}$ adsorption on $d h$ - $\mathrm{BN}$ with $\mathrm{B}_{\mathrm{N}}$, one hydrogen prefers to adsorb on the central boron atom (B4) whereas the other prefers to stay at the bridge between B1 and B4, as shown in Fig. $4 \mathrm{~d}$. The adsorption energy of this configuration is $-1.51 \mathrm{eV}$.

Charge density difference (CDD) plots, shown in the bottom panels of Fig. 4, indicate noticeable rearrangement of electron density and electron transfer. For $\mathrm{V}_{\mathrm{N}}$ and $\mathrm{B}_{\mathrm{N}}$, the $d h$-BN donates 1.168 and 1.098 electrons to the atomic hydrogens, respectively. For $\mathrm{V}_{\mathrm{B}}$, the defect gains 0.902 electrons from atomic hydrogens. In the case of $\mathrm{SW}, 0.120$ electrons transfer from $d h$-BN to

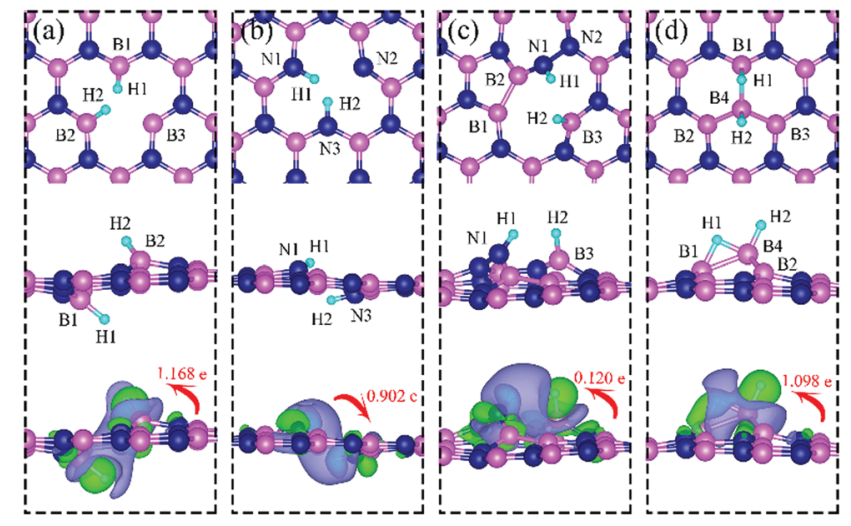

Fig. 4 Optimized structures of two hydrogen atoms adsorbed at energetically favorable sites on $d h-B N$ with (a) $V_{N}$, (b) $V_{B}$, (c) $S W$, and (d) $B_{N}$. The magenta, blue, cyan balls represent $\mathrm{B}, \mathrm{N}$ and $\mathrm{H}$ atoms, respectively. The top and middle panel show the top and side views, respectively. Bottom panel: Corresponding side view of geometric structure with charge density difference plot. The green and violet isosurfaces with isovalue of \pm 0.003 e $\AA^{-3}$, represent accumulation and depletion of charge, respectively. The red arrow shows the direction of electron transfer evaluated with Bader charge analysis. 
Table 1 The adsorption energy without $\left(E_{\text {ads }}\right)$ and with $\left(E_{\text {ads }}{ }^{\prime}\right)$ ZPE correction, geometrical parameters, and notable frequencies for adsorption of 2 atomic Hydrogen on $d h-B N$

\begin{tabular}{lllllll}
\hline Defects & Sites & $E_{\text {ads }}(\mathrm{eV})$ & $E_{\text {ads }}{ }^{\prime}(\mathrm{eV})$ & Bond lengths $(\AA)$ & Notable frequencies $\left(\mathrm{cm}^{-1}\right)$ \\
\hline $\mathrm{V}_{\mathrm{N}}$ & $\mathrm{B} 1, \mathrm{~B} 2$ & -1.61 & -1.28 & $d_{(\mathrm{H}-\mathrm{H})}=3.087, d_{(\mathrm{H} 1-\mathrm{B} 1)}=1.189, d_{(\mathrm{H} 2-\mathrm{B} 2)}=1.189$ & $2557.0 \mathrm{~cm}^{-1}, 2560.8 \mathrm{~cm}^{-1}$ \\
$\mathrm{~V}_{\mathrm{B}}$ & $\mathrm{N} 1, \mathrm{~N} 2$ & -4.74 & -4.22 & $d_{(\mathrm{H}-\mathrm{H})}=1.869, d_{(\mathrm{H} 1-\mathrm{N} 1)}=1.012, d_{(\mathrm{H} 2-\mathrm{N} 2)}=1.009$ & $3458.8 \mathrm{~cm}^{-1}, 3507.7 \mathrm{~cm}^{-1}$ \\
$\mathrm{SW}$ & $\mathrm{B} 3, \mathrm{~N} 1$ & 1.16 & 1.41 & $d_{(\mathrm{H}-\mathrm{H})}=1.687, d_{(\mathrm{H} 1-\mathrm{N} 1)}=1.004, d_{(\mathrm{H} 2-\mathrm{B} 3)}=1.190, d_{(\mathrm{B} 3-\mathrm{N} 1)}=2.632$ & $2543.6 \mathrm{~cm}^{-1}, 3572.5 \mathrm{~cm}^{-1}$ \\
$\mathrm{~B}_{\mathrm{N}}$ & $\mathrm{B} 1, \mathrm{~B} 4$ & -1.51 & -1.32 & $d_{(\mathrm{H}-\mathrm{H})}=1.757, d_{(\mathrm{H} 2-\mathrm{B} 4)}=1.207, d_{(\mathrm{H} 1-\mathrm{B} 4)}=1.274, d_{(\mathrm{H} 1-\mathrm{B} 1)}=1.315, d_{(\mathrm{B} 1-\mathrm{B} 4)}=1.858$ & $2052.5 \mathrm{~cm}^{-1}, 2440.5 \mathrm{~cm}^{-1}$
\end{tabular}

atomic hydrogens. We also find that adsorption of $2 \mathrm{H}$ affects electronic density of states of $d h$-BN (Fig. 5) in different ways depending on the defect type. For instance, comparison of Fig. 2a with Fig. 5a shows that $2 \mathrm{H}$ adsorption increases the HOMO-LUMO gap for $d h$-BN with $\mathrm{V}_{\mathrm{N}}$. For $\mathrm{V}_{\mathrm{B}}$ type defect, Fig. $5 \mathrm{~b}$ attests to the disappearance of the defect states near the Fermi level (see Fig. 2b) on $2 \mathrm{H}$ adsorption, leaving only the mid-gap state with spin down channel now drawn closer to the valence band, as compared to pristine $\mathrm{V}_{\mathrm{B}}$. For the case of SW, adsorption of $2 \mathrm{H}$ leads to only a slight change of the HOMO-LUMO gap. Finally, for the case of $\mathrm{B}_{\mathrm{N}}$, adsorption of $2 \mathrm{H}$ widens the HOMO-LUMO gap, such that HOMO and LUMO lie at the top of the valence and bottom of conduction bands, respectively. Overall, our calculations indicate that hydrogen atoms adsorb most strongly on $d h$-BN with a $\mathrm{V}_{\mathrm{B}} \operatorname{defect}\left(E_{\mathrm{ads}}=-4.74 \mathrm{eV}\right)$ and not at all when the defect is SW $\left(E_{\text {ads }}=+1.04 \mathrm{eV}\right)$. The inclusion of zero-point energy correction changes the binding energy of $2 \mathrm{H}$ to $d h$-BN to $-1.28,-4.22,+1.41$, and $-1.32 \mathrm{eV}$ with $\mathrm{V}_{\mathrm{N}}, \mathrm{V}_{\mathrm{B}}$, $\mathrm{SW}$, and $\mathrm{B}_{\mathrm{N}}$, respectively. The adsorption of $\mathrm{H}$ on $d h$-BN creates either $\mathrm{B}-\mathrm{H}, \mathrm{N}-\mathrm{H}$ bonds, or both. The vibrational frequencies of the stretching mode of the bonds should serve as an identifier
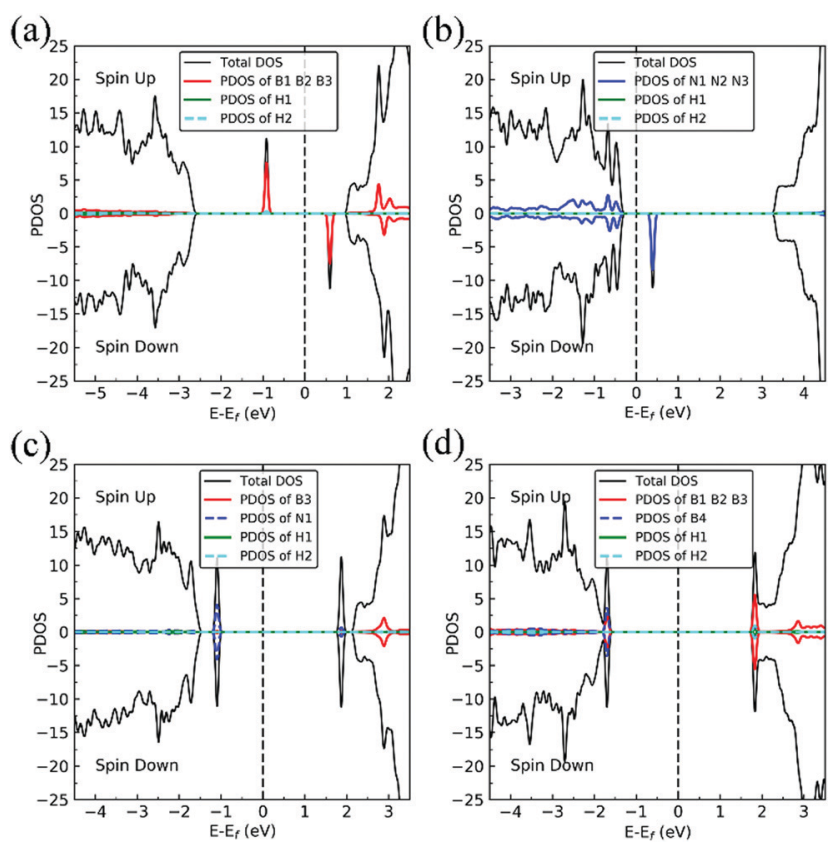

(d)

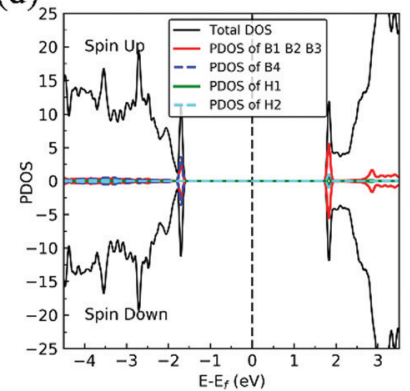

Fig. 5 Spin-polarized total density of states (TDOS) (black line), projected density of states (PDOS) in defect regions (red or blue) and spin-polarized PDOS of adsorbed hydrogens (green and cyan) on $2 \mathrm{H}-d h-\mathrm{BN}$ with (a) $\mathrm{V}_{\mathrm{N}}$, (b) $V_{B}$, (c) $S W$, and (d) $B_{N}$, respectively. The position of the Fermi level $\left(E_{F}\right)$ is indicated by dashed line.

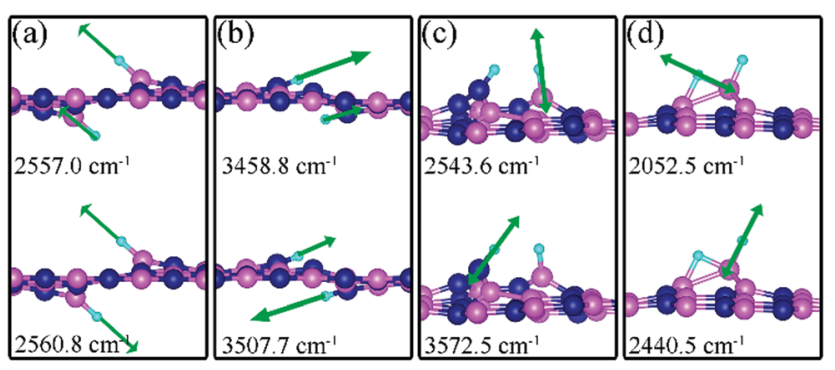

Fig. 6 Notable displacement patterns of two hydrogen atoms adsorbed on dh-BN with (a) $V_{N}$, (b) $V_{B}$, (c) SW, and (d) $B_{N}$ defect. The magenta, blue, black and light blue balls represent the $\mathrm{B}, \mathrm{N}$, and $\mathrm{H}$ atoms, respectively. Arrows indicate displacement directions.

of the defect sites to which $\mathrm{H}$ adsorbs. The notable vibrational frequencies of such modes are listed in Table 1 and their corresponding vibrational patterns are shown in Fig. 6.

\section{CO on $d h$-BN}

Carbon monoxide (CO) is one of the major common toxic gases in the atmosphere and is the main component of syngas, the essential reactant for (higher) alcohol synthesis. Fig. 7 shows the geometric structure of the lowest energy configuration of $\mathrm{CO}$ adsorbed on $\mathrm{V}_{\mathrm{N}}, \mathrm{V}_{\mathrm{B}}$, SW, and $\mathrm{B}_{\mathrm{N}}$. Table 2 summarizes the binding energy, geometric parameters, and notable frequencies for CO binding on the four types of defects in $h$-BN.

\section{$\mathrm{CO}$ adsorption on $\mathrm{V}_{\mathrm{N}}$}

On $\mathrm{V}_{\mathrm{N}}$, CO binds at the defect sites with the $\mathrm{O}$ atom bonded to $\mathrm{B} 1$ and the $\mathrm{C}$ atom bonded to B2 and B3, as shown in Fig. 7a, in agreement with prior results. ${ }^{43,44}$ The adsorption causes out-ofplane displacement of the B1 atom by about $1 \AA$, which is quite large. The CO bond length increases from $1.134 \AA$ (gas phase) to $1.403 \AA$ (adsorbed), while $d_{(\mathrm{C}-\mathrm{B} 2)}\left(d_{(\mathrm{C}-\mathrm{B} 3)}\right)$ and $d_{(\mathrm{O}-\mathrm{B})}$ are calculated to be 1.528 and $1.417 \AA$, respectively. The CO bond length of $1.403 \AA$ suggests that a bond order of 1 , i.e. adsorption causes scission of the two $\pi$-bonds of the CO molecule, making it ready for a further reaction such as dissociation or hydrogenation. The adsorption energy of $\mathrm{CO}$ on $d h-\mathrm{BN}\left(\mathrm{V}_{\mathrm{N}}\right)$ is $-3.45 \mathrm{eV}$, which is not far from $-4.04 \mathrm{eV}$ or $-3.97 \mathrm{eV}$ reported elsewhere. ${ }^{43,44}$ The adsorption energy is $-3.22 \mathrm{eV}$ with ZPE correction. The Bader charge analysis shows that 2.070 electrons are transferred from $d h$-BN to the CO molecule. As shown in the bottom panel of Fig. 7a, a sizable electron density accumulation (green region) appears between $\mathrm{B}$ and $\mathrm{C}$, and between $\mathrm{B}$ and $\mathrm{O}$, which verifies formation of the $\mathrm{B}-\mathrm{C}$ and $\mathrm{B}-\mathrm{O}$ bonds. A loss of electron density (violet region) is shown around the $\mathrm{C}-\mathrm{O}$ bond, 


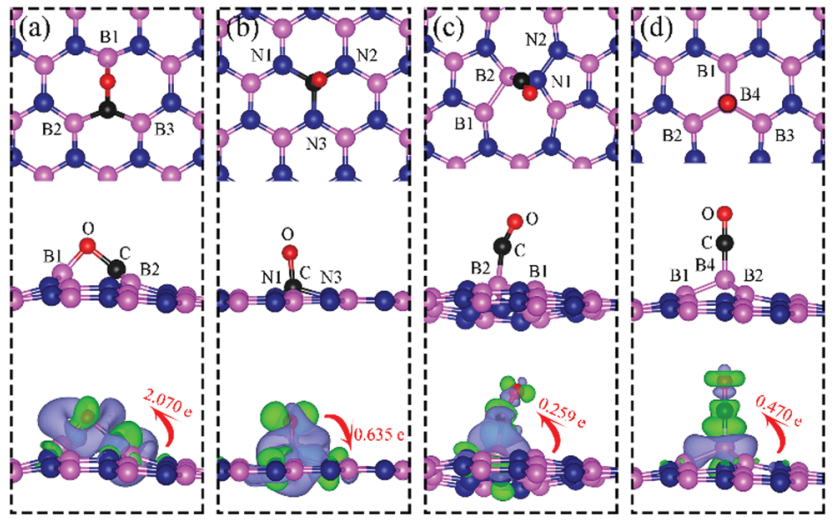

Fig. 7 Top and side views of the most energetically favorable configurations of adsorbed CO on dh-BN with (a) $V_{N}$, (b) $V_{B}$, (c) SW, and (d) $B_{N}$ type defect. The magenta, blue, black and red balls represent the $B, N, C$, and $O$ atoms, respectively. The top and middle panel shows the top and side views, respectively. Bottom: The corresponding side views of the geometric structures with the charge density difference. The green and violet isosurfaces with isovalue of \pm 0.003 e $\AA^{-3}$, represent the accumulation and depletion of electron, respectively. The red arrow shows the electron transfer in direction and value using Bader charge analysis.

confirming that adsorption weakens the $\mathrm{C}-\mathrm{O}$ bond by breaking its two $\pi$-bonds. In Fig. 8a, which contains PDOS of CO adsorption on $d h-\mathrm{BN}\left(\mathrm{V}_{\mathrm{N}}\right)$, the orbital mixing between the occupied states of $\mathrm{CO}$ molecules (in red line) and the states of $\mathrm{B}$ atoms (around the vacancy in blue line). This obvious overlap of states of $\mathrm{CO}$ and $\mathrm{V}_{\mathrm{N}}$ indicates the strong chemisorption of the CO molecule caused by dangling bonds of the $\mathrm{B}$ atoms around the vacancy site. In addition, the peaks in the density of states shift toward the higher energy since a charge transfer takes place from the $d h$-BN surface to the CO molecule.

The strong adsorption of $\mathrm{CO}$ on $\mathrm{V}_{\mathrm{N}}$ with both $\mathrm{C}$ and $\mathrm{O}$ bond to Boron atoms of $d h$-BN significantly affects the CO stretching mode. We found that its frequency is $1069.2 \mathrm{~cm}^{-1}$, as compared to that of $2115.0 \mathrm{~cm}^{-1}$ of the isolated CO molecule. This is a large shift and reflects the strong bond of $\mathrm{CO}$ with the support. In addition, we found that there are several vibrational modes that show a strong coupling between $\mathrm{CO}$ and $d h$-BN whose frequencies are listed in Table 2 and whose vibrational patterns are shown in Fig. 9.

\section{CO adsorption on $V_{B}$}

The most stable adsorption configuration of $\mathrm{CO}$ adsorption on $\mathrm{V}_{\mathrm{B}}$ is shown in Fig. 7b. In this configuration, CO binds to N1, $\mathrm{N} 2$ and $\mathrm{N} 3$ atoms in a vertical orientation via $\mathrm{C}-\mathrm{N}$ bond, which is similar to that previously reported. ${ }^{43,44} \mathrm{C}-\mathrm{O}$ bond length (a)

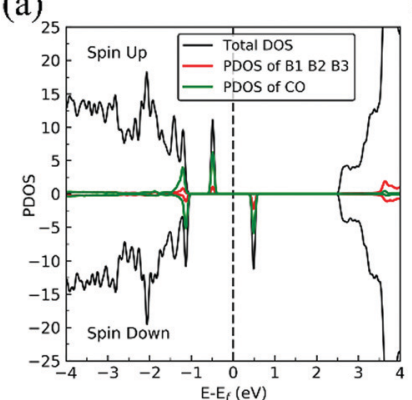

(c)

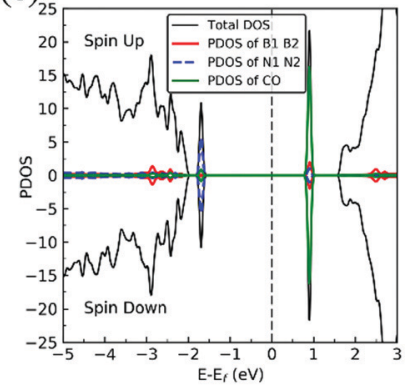

(d)
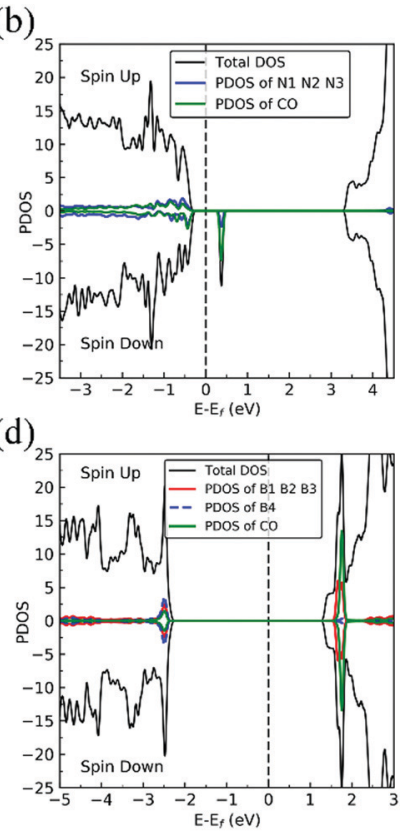

Fig. 8 Spin-polarized TDOS (black line), PDOS of defect area (red or blue) and PDOS of adsorbed CO (green line) of CO-dh-BN with (a) $V_{N}$, (b) $V_{B}$, (c) $S W$, and (d) $B_{N}$, respectively. The position of the Fermi level $\left(E_{F}\right)$ is indicated by dashed line.

increases from $1.134 \AA$, in gas phase CO, to $1.387 \AA$, while $d_{(\mathrm{C}-\mathrm{N})}$ is found to be $1.495 \AA$. The CO bond length is larger than that of a typical bond order 2, but smaller than that of bond order 1 . The calculated adsorption energy of $-4.70 \mathrm{eV}(-4.45 \mathrm{eV}$ with ZPE correction) is smaller than that $(-5.63$ or $-5.86 \mathrm{eV})$ reported elsewhere. ${ }^{43,44}$ The difference may arise from the form of the exchange-correlation functional used in the DFT calculations or from differences in the geometry of the reference structure, as we discuss below. The Bader charge analysis shows that about 0.635 electrons are transferred from the $\mathrm{CO}$ molecule to the support. In the bottom panel of Fig. $7 \mathrm{~b}$, a sizable electron density accumulation (green region) appeared between the $\mathrm{C}$ and $\mathrm{N}$ atoms, which affirms the strong interaction between the $\mathrm{CO}$ molecule and $\mathrm{N}$ atoms around the vacancy. A loss of electron density (violet region) surrounds the region between $\mathrm{C}$ and $\mathrm{O}$ atoms, which shows a weakened $\mathrm{C}-\mathrm{O}$ bond. In Fig. 8b, PDOS of CO adsorption on $d h-\mathrm{BN}$ at $\mathrm{V}_{\mathrm{B}}$ around the Fermi level shows orbital mixing between the occupied states of the $\mathrm{CO}$ molecule and orbitals of B atoms, indicating chemisorption of $\mathrm{CO}$ on $d h$-BN.

As for $\mathrm{CO}$ on $\mathrm{V}_{\mathrm{N}}$, the strong adsorption of $\mathrm{CO}$ on $\mathrm{V}_{\mathrm{B}}$ significantly affects the $\mathrm{CO}$ stretching mode. We found that

Table 2 The adsorption energy without $\left(E_{\text {ads }}\right)$ and with $\left(E_{\text {ads }}{ }^{\prime}\right)$ ZPE correction, geometrical parameters, and notable frequencies for adsorption of $C O$ on $d h$-BN. Note that the frequency of the CO stretch mode is $2115.0 \mathrm{~cm}^{-1}$ in the gas phase

\begin{tabular}{|c|c|c|c|c|}
\hline Defects & $E_{\text {ads }}(\mathrm{eV})$ & $E_{\text {ads }}{ }^{\prime}(\mathrm{eV})$ & Bond length $(\AA)$ and Bond angle $\left(^{\circ}\right)$ & Notable frequencies $\left(\mathrm{cm}^{-1}\right)$ \\
\hline $\mathrm{V}_{\mathrm{B}}$ & -4.70 & -4.45 & $d_{(\mathrm{C}-\mathrm{O})}=1.387, d_{(\mathrm{C}-\mathrm{N} 1)}=1.495, \theta_{(\mathrm{O}-\mathrm{C}-\mathrm{N} 1)}=110.8^{\circ}$ & $140.2,147.6,903.6,973.8,981.7$ \\
\hline SW & 0.42 & 0.46 & $d_{(\mathrm{C}-\mathrm{O})}=1.150, d_{(\mathrm{C}-\mathrm{B} 2)}=1.631, \theta_{(\mathrm{O}-\mathrm{C}-\mathrm{B} 2)}=159.0^{\circ}$ & $64.0,247.7,398.6,1988.3$ \\
\hline
\end{tabular}




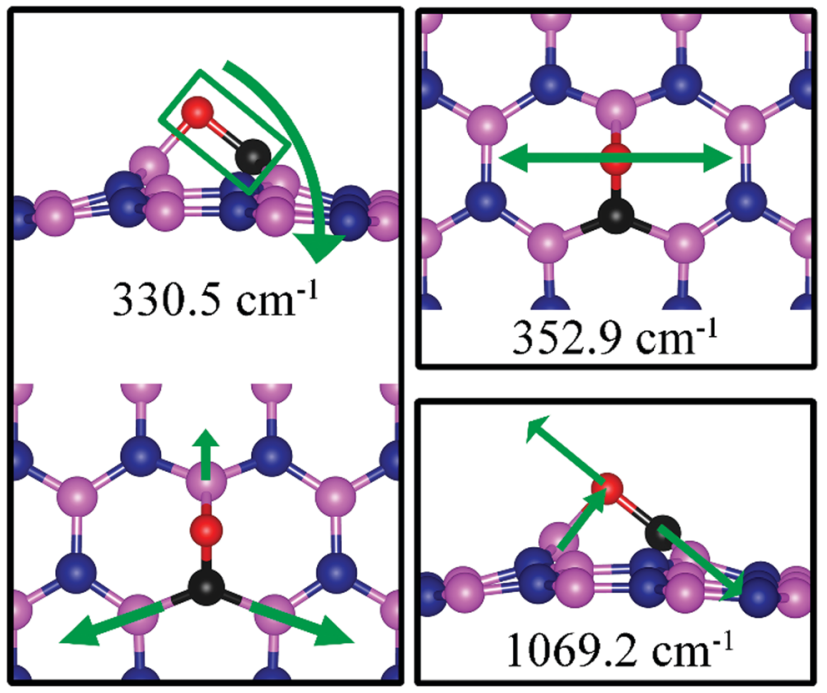

Fig. 9 Notable vibrational modes of $\mathrm{CO}$ adsorbed on $d h-\mathrm{BN}$ with $\mathrm{V}_{\mathrm{N}}$. The magenta, blue, black and red balls represent the $B, N, C$, and $O$ atoms, respectively. Arrows indicate vibrational patterns.

its frequency is $903.6 \mathrm{~cm}^{-1}$, as compared to $2115.0 \mathrm{~cm}^{-1}$ of the isolated CO molecule. Such large change is consistent with the elongation of $\mathrm{CO}$ bond length upon adsorption. In addition, we found several vibrational modes that show strong coupling between $\mathrm{CO}$ and $d h$-BN, whose frequencies are listed in Table 2 and whose displacement patterns are illustrated in Fig. 10.

\section{CO adsorption on SW}

The most energetically favorable configuration of CO adsorption on SW is shown in Fig. 7c. Here CO adsorbs at the B site by pulling the $\mathrm{B}$ atom out from the $d h$ - $\mathrm{BN}$ plane, resulting in $\mathrm{C}-\mathrm{O}$ bond length of $1.150 \AA$ and $\mathrm{C}-\mathrm{B}$ bond length of $1.631 \AA$. The adsorption energy of $\mathrm{CO}$ is found to be $0.42 \mathrm{eV}(0.46 \mathrm{eV}$ with ZPE correction), which is significantly different from $-0.58 \mathrm{eV}$ reported in a previous study. ${ }^{45}$ While both studies show similar adsorption configuration, the large difference in binding energies is puzzling, even after accounting for differences in the exchange correlation functional used in DFT calculations. One plausible reason for such discrepancy could be how the reference system for calculating binding energy, i.e., $h$-BN with SW defect, was created and optimized. We performed tests for couple of scenarios. For example, if the reference clean SW defect system is flat and without minimized stress, its energy is $0.87 \mathrm{eV}$ higher than the clean SW defect system we use, which is stress free and not flat. Secondly, if the reference system is prepared as a non-flat sheet but stress is not minimized, its energy is $0.39 \mathrm{eV}$ higher than ours. If such higher energy structures of clean SW defect system were used in ref. 45, it would boost the binding energy of $\mathrm{CO}$ significantly as such adsorption would break symmetry making the $d h$-BN non-flat or creating perturbation to release stress. Moreover, our result of positive binding energy, i.e., endothermic adsorption, of CO molecule is reasonable because $\mathrm{B}$ atom has 3 valence electrons and prefers to make 3 covalent bonds. In the SW defect, it

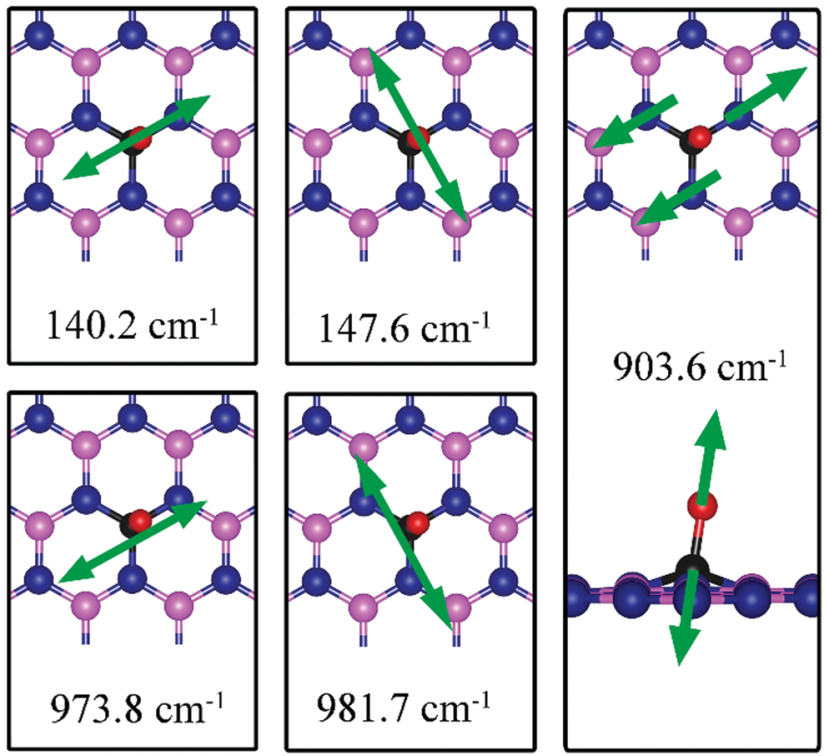

Fig. 10 Notable vibrational modes of $\mathrm{CO}$ adsorption on $d h-\mathrm{BN}$ with $\mathrm{V}_{\mathrm{B}}$. The magenta, blue, black and red balls represent the $B, N, C$, and $O$ atoms, respectively. Arrows indicate vibrational patterns.

already has 3 covalent bonds. Making another covalent bond with the adsorbed $\mathrm{CO}$ molecule is thus not energetically favorable. A similar rationale led to the conclusion that $\mathrm{CO}$ does not chemically bind to SW in BN nanotube. ${ }^{46}$ To further validate our results, we calculate the physisorption energy of $\mathrm{CO}$ on SW to find that it is $-0.14 \mathrm{eV}$, indicating $\mathrm{CO}$ preference to be in the physisorbed state. We also performed Nudged Elastic Band ${ }^{47,48}$ calculations between the physisorbed and chemisorbed configurations (Fig. S1 in ESI $\dagger$ ) and found that there is a barrier of $0.57 \mathrm{eV}$ for the physisorbed molecule to adsorb chemically on the SW defect and a barrier of $0.01 \mathrm{eV}$ for the chemisorbed $\mathrm{CO}$ to desorb. The latter together with the positive binding energy indicate a weak chemisorption of the $\mathrm{CO}$ molecule at SW defect. Bader charge analysis of chemisorbed $\mathrm{CO}$ on SW shows 0.259 electrons transferred from the surface to CO. The charge density difference plot of $\mathrm{CO}$ on $d h$-BN (SW) as shown in the bottom panel of Fig. 7c indicates a sizable electron density accumulation (green region) between the $\mathrm{B}$ and $\mathrm{C}$ atoms, consistent with the formation of $\mathrm{B}-\mathrm{C}$ bond. However, the $\mathrm{B}-\mathrm{C}$ bond length is $1.631 \AA$, longer than that for $\mathrm{CO}$ on $\mathrm{V}_{\mathrm{N}}$ and $\mathrm{V}_{\mathrm{B}}$ defects, indicating that the strength of CO binding on $d h$-BN with SW is weaker than that for the two other defects, consistent with calculated positive adsorption energies. The PDOS in Fig. 8c shows that the conduction band moves toward lower energy and that the valence band shifts toward higher energy, reducing the band gap of $d h$-BN, albeit the energy gap is still large and there is no available electronic state that is at or close to the Fermi level.

As the strength of adsorption of $\mathrm{CO}$ on $\mathrm{SW}$ is much reduced compared to that on $\mathrm{V}_{\mathrm{B}}$ and $\mathrm{V}_{\mathrm{N}}$, the CO stretching frequency is found to be $1988.3 \mathrm{~cm}^{-1}$. This mode is softer than that in isolated $\mathrm{CO}$ molecule due to the reduction in strength of $\mathrm{CO}$ bond and its elongation upon adsorption. In addition, we find several vibrational modes that show strong contribution from $\mathrm{C}$ 
and $\mathrm{O}$ movements whose frequencies are listed in Table 2 and whose displacement patterns are shown in Fig. 11.

\section{CO adsorption on $\mathrm{B}_{\mathrm{N}}$}

Fig. 7d shows the energetically favored CO adsorption configuration on $d h$ - $\mathrm{BN}$ with $\mathrm{B}_{\mathrm{N}}$, in which $\mathrm{CO}$ binds perpendicularly on top of the central B atom (labeled as B4) with an adsorption energy of $-2.53 \mathrm{eV}(-2.43 \mathrm{eV}$ with $\mathrm{ZPE}$ correction). The calculated bond length $d_{(\mathrm{C}-\mathrm{B})}$ and $d_{(\mathrm{C}-\mathrm{O})}$ are $1.444 \AA$ and $1.163 \AA$ (elongated by $\sim 0.03 \AA$ from that in gas-phase), respectively. CDD plot of $\mathrm{CO}$ on $d h$-BN with $\mathrm{B}_{\mathrm{N}}$ shown in the bottom panel of Fig. 7d indicates a sizable electron density accumulation (green region) between the $\mathrm{B}$ and $\mathrm{C}$ atoms, confirming the strong interaction between the CO molecule and its neighboring $\mathrm{B}$ atoms around the defect area. A small loss of electron density (violet region) is seen over the $\mathrm{C}-\mathrm{O}$ bond, indicating weakening of the $\mathrm{C}-\mathrm{O}$ bond, consistent with its elongation (from $1.134 \AA$ in gas phase to $1.163 \AA$ ). A large loss of electron density (violet region) is found over the $\mathrm{B} 4-\mathrm{B} 1, \mathrm{~B} 4-\mathrm{B} 2$ and $\mathrm{B} 4-\mathrm{B} 3$ bonds, suggesting that the bonds are weakened on $\mathrm{CO}$ adsorption. Bader charge analysis shows a net 0.470 electrons transferred from $d h$-BN (with $\mathrm{B}_{\mathrm{N}}$ ) to CO. After $\mathrm{CO}$ adsorption on $d h$-BN with $\mathrm{B}_{\mathrm{N}}$, the peaks in density of states shift toward lower energy, as shown in Fig. 8d. More importantly, the defect states merge with the valence and conduction bands, suggesting the absence of dangling bonds and the inability of more molecules to adsorb, thus passivating the catalytic activity of $d h$-BN with $\mathrm{B}_{\mathrm{N}}$.
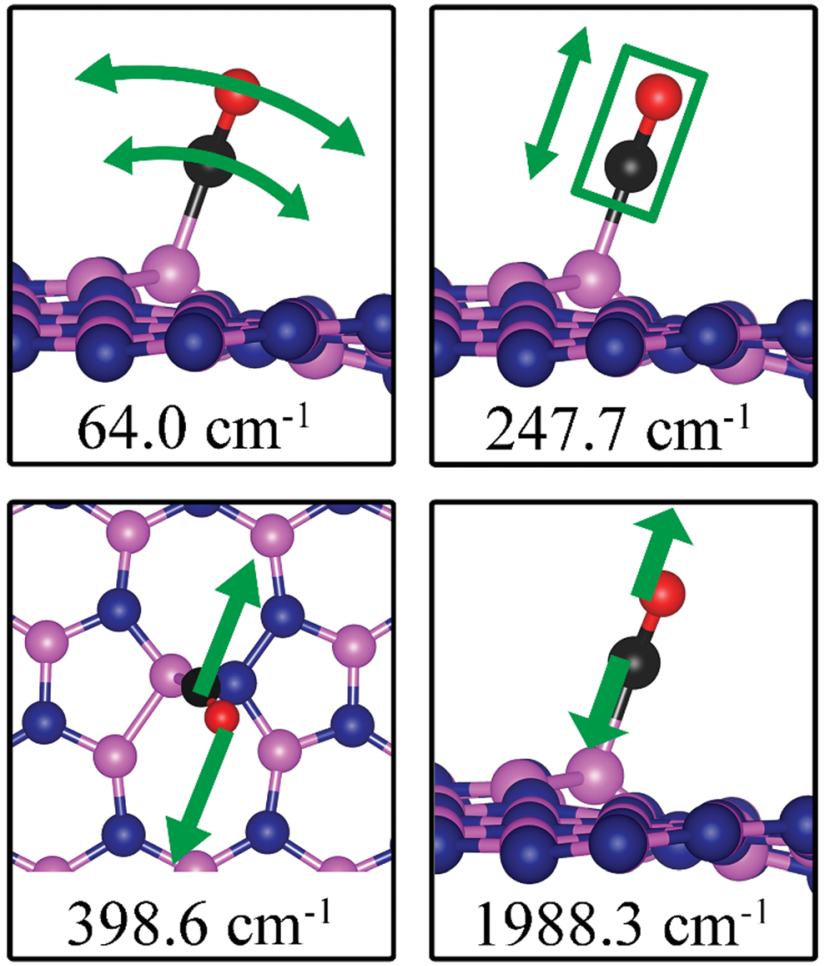

Fig. 11 Notable vibrational modes of $\mathrm{CO}$ adsorption on dh-BN with SW defect. The magenta, blue, black and red balls represent the $B, N, C$, and $O$ atoms, respectively. Arrows indicate vibrational patterns. The rectangular encloses the atoms that concertedly move.
The stretching frequency of the adsorbed $\mathrm{CO}$ molecule on dh-BN with $\mathrm{B}_{\mathrm{N}}$ is found to be $1999.2 \mathrm{~cm}^{-1}$. This mode is slightly softer than that in isolated $\mathrm{CO}$ molecule commensurate with small reduction in strength of the $\mathrm{CO}$ bond as a result of slight elongation upon adsorption. Here again we find several vibrational modes that show strong coupling between $\mathrm{CO}$ and $d h$-BN, displacement patterns of six of which are shown in Fig. 12.

\section{$\mathrm{CO}_{2}$ on $d h-\mathrm{BN}$}

The geometric structure of $\mathrm{CO}_{2}$ adsorption on $\mathrm{V}_{\mathrm{N}}, \mathrm{V}_{\mathrm{B}}$, SW, and $B_{N}$ are shown in Fig. 13. The corresponding adsorption energies and geometric parameters are summarized in Table 3.

\section{$\mathrm{CO}_{2}$ adsorption on $\mathrm{V}_{\mathrm{N}}$}

On $d h$-BN with $\mathrm{V}_{\mathrm{N}}, \mathrm{CO}_{2}$ binds at the defect site, with an adsorption energy of $-1.83 \mathrm{eV}(-1.72 \mathrm{eV}$ with $\mathrm{ZPE}$ correction), indicating a strong chemisorption of the $\mathrm{CO}_{2}$ molecule at the vacancy site. As shown in Fig. 13a, the adsorption pulls B1, B2 and $\mathrm{B} 3$ atoms slightly out of the $d h$-BN plane and the $\mathrm{CO}_{2}$ molecule lies in the proximity of three boron sites near the vacancy with bond lengths, $d_{(\mathrm{O} 1-\mathrm{B} 1)}$, and $d_{(\mathrm{C}-\mathrm{B} 2)}\left(d_{(\mathrm{C}-\mathrm{B} 3)}\right)$, of 1.382 and $1.672 \AA$, respectively. The molecule is bent with the O1-C-O2 angle of $113.7^{\circ}$. The bond lengths, $d_{(\mathrm{C}-\mathrm{O} 1)}$ and $d_{(\mathrm{C}-\mathrm{O} 2)}$, are 1.438 and $1.311 \AA$, respectively (both are longer than that of $1.172 \AA$ of gas phase $\mathrm{CO}_{2}$ ), indicating that the original $\mathrm{C}-\mathrm{O}$ bonds are stretched upon interaction between $\mathrm{CO}_{2}$ and $d h$-BN with $\mathrm{V}_{\mathrm{N}}$. Bader charge analysis shows that about 2.002 electrons
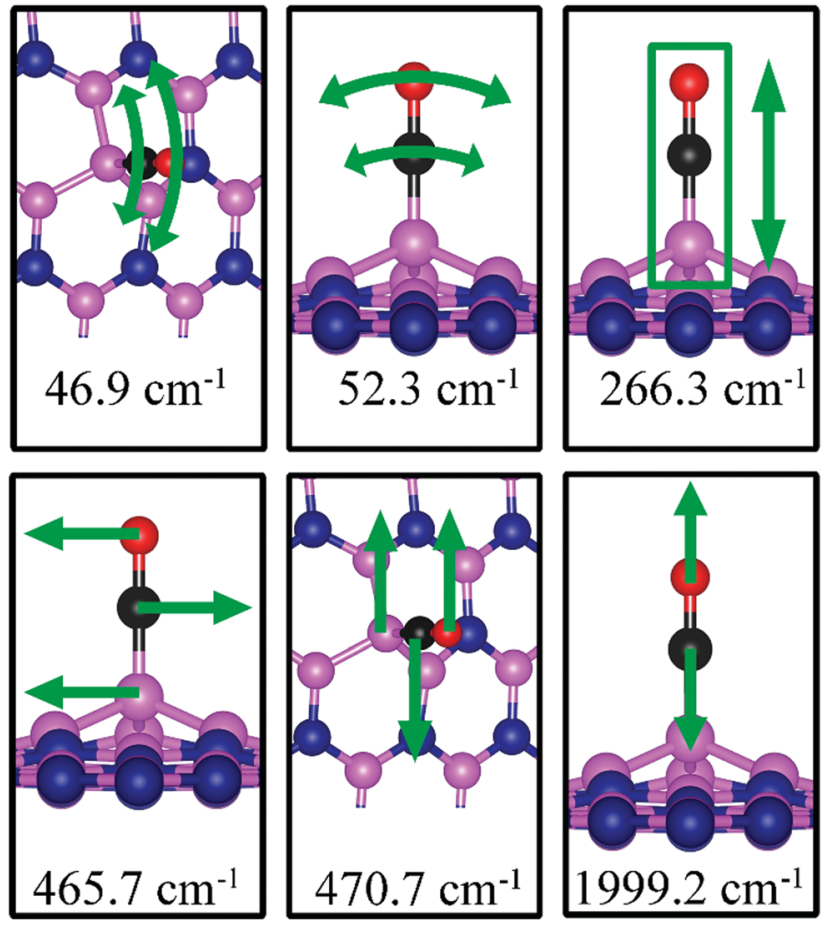

Fig. 12 Notable vibrational modes of $\mathrm{CO}$ adsorption on $d h-\mathrm{BN}$ with $\mathrm{B}_{\mathrm{N}}$. The magenta, blue, black and red balls represent the $B, N, C$, and $O$ atoms, respectively. Arrows indicate vibrational patterns. The rectangular encloses the atoms that are concertedly move together. 


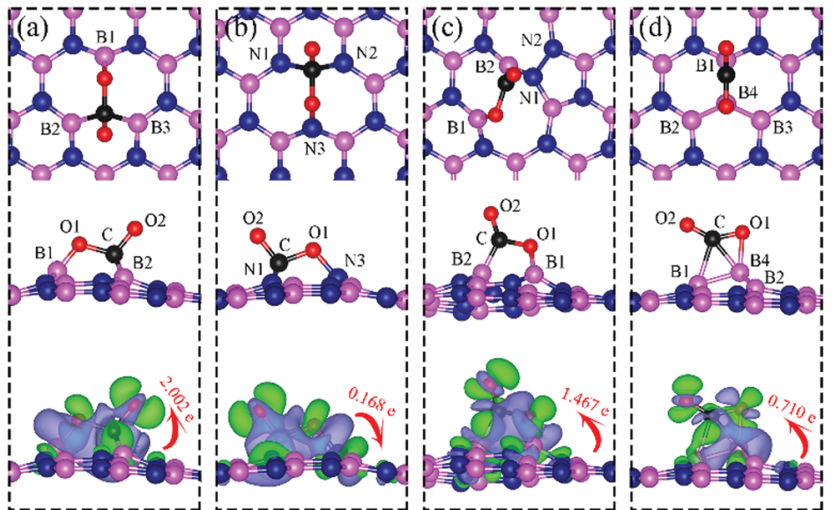

Fig. 13 Top and side views of the most energetically favorable configurations of adsorbed $\mathrm{CO}_{2}$ on the $d h-B N$ with (a) $V_{N}$, (b) $V_{B}$, (c) $S W$, and (d) $B_{N}$. The magenta, blue, black and red balls represent the B, N, C, and $O$ atoms, respectively. The top and middle panel shows the top and side views, respectively. Bottom: The corresponding side views of the geometric structures with the charge density difference plot. The green and violet isosurfaces with isovalue of \pm 0.003 e $\AA^{-3}$, represent the accumulation and depletion of electron, respectively. The red arrow shows the electron transfer in direction and value.

transferred from $d h$-BN to the $\mathrm{CO}_{2}$ molecule. CDD plot of $\mathrm{CO}_{2}$ on $d h$-BN with $\mathrm{V}_{\mathrm{N}}$, shown in the bottom panel of Fig. 13a, indicates a sizable electron density accumulation (green region) appearing between the B2 (B3) and C atoms and between the B1 and $\mathrm{O}$ atoms, consistent with the formation of new bonds B2(B3)-C and B1-C. A loss of electron density (violet region) between $\mathrm{C}-\mathrm{O}$ is also consistent with the elongation and weakening of the $\mathrm{C}-\mathrm{O}$ bond. PDOS shown in Fig. 14a indicates overlaps, i.e., hybridizations, between states of $\mathrm{CO}_{2}$ molecule and the states of $\mathrm{B}$ atoms, indicating the chemisorption of $\mathrm{CO}_{2}$ on the $d h-\mathrm{BN}$ with $\mathrm{V}_{\mathrm{N}}$ and the strong interaction between the molecule and the $d h$-BN sheet. We also find that the defect states, i.e., the ones near the Fermi level, are mostly contributed from $\mathrm{CO}_{2}$ and shifted toward lower energy, closer to VBM. This phenomenon indicates that the defect states have migrated (transferred) to the adsorbed $\mathrm{CO}_{2}$ molecules.

Since $\mathrm{CO}_{2}$ adsorbs on $d h$-BN with a bent configuration, the normal vibrational modes of $\mathrm{CO}_{2}$ molecule (symmetric stretching, antisymmetric stretching, bending) are absent. Instead, we found few notable vibrational modes that include contributions from the adsorbed $\mathrm{CO}_{2}$ molecules, as shown in Fig. 15, with their frequencies listed in Table 3. Among them, the CO stretching (a)

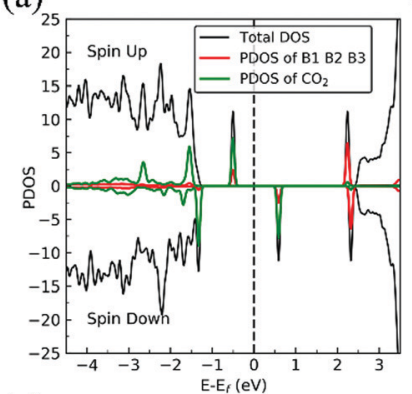

(c)

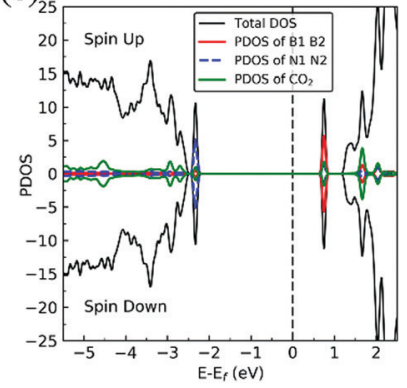

(d)
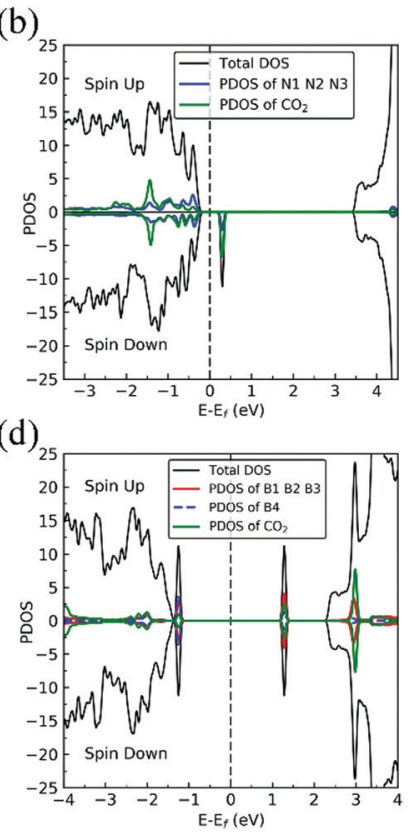

Fig. 14 Spin-polarized total density of states (TDOS) (black line), and spinpolarized PDOS of defect area (red line or blue line) and of adsorbed $\mathrm{CO}_{2}$ (green line) of $\mathrm{CO}_{2}-d h-\mathrm{BN}$ with (a) $\mathrm{V}_{\mathrm{N}}$, (b) $\mathrm{V}_{\mathrm{B}}$, (c) SW, and (d) $\mathrm{B}_{\mathrm{N}}$, respectively. The position of the Fermi level $\left(E_{F}\right)$ is indicated by dashed line.

mode is of particular interest as it is infra-red active and its frequency is $1194.0 \mathrm{~cm}^{-1}$, which is different from that $\left(1069.2 \mathrm{~cm}^{-1}\right)$ of the adsorbed $\mathrm{CO}$ molecule on the same defect.

\section{$\mathrm{CO}_{2}$ adsorption on $\mathrm{V}_{\mathrm{B}}$}

On $d h$ - $\mathrm{BN}$ with $\mathrm{V}_{\mathrm{B}}, \mathrm{CO}_{2}$ binds at the $\mathrm{N}$ sites with an adsorption energy of $-0.75 \mathrm{eV}(-0.59 \mathrm{eV}$ with ZPE correction). Fig. 13b shows the corresponding adsorption configuration, close to that reported in previous work, ${ }^{49}$ which also reported a binding energy of $-0.62 \mathrm{eV}$. In this configuration, $\mathrm{CO}_{2}$ lies in between the three $\mathrm{N}$ sites with bond lengths, $d_{(\mathrm{C}-\mathrm{N} 1)}$ and $d_{(\mathrm{O} 1-\mathrm{N} 3)}$, of $1.496 \AA$ and $1.446 \AA$, respectively. The bond lengths, $d_{(\mathrm{C}-\mathrm{O} 1)}$ and $d_{(\mathrm{C}-\mathrm{O} 2)}$, are 1.409 and $1.353 \AA$ (longer than that $1.172 \AA$ of an isolated molecule), respectively, indicating weakening of the $\mathrm{C}-\mathrm{O}$ bonds. The Bader charge analysis shows 0.168 electrons transferred from the molecule to the $d h-\mathrm{BN}$. CDD plot of $\mathrm{CO}_{2}$ on $d h$-BN with $\mathrm{V}_{\mathrm{B}}$ shown in Fig. 13b shows a sizable electron density accumulation (green region) between the $\mathrm{N}$ and $\mathrm{C}$ atoms, confirming the formation of $\mathrm{N}-\mathrm{C}$ bond, and a loss of

Table 3 The adsorption energy without $\left(E_{\text {ads }}\right)$ and with $\left(E_{\text {ads }}{ }^{\prime}\right)$ ZPE correction and geometrical parameters for adsorption of $\mathrm{CO}_{2}$ on $d h-\mathrm{BN}$. Note that the frequency of the $\mathrm{CO}$ stretch mode is $2115.0 \mathrm{~cm}^{-1}$ in the gas phase

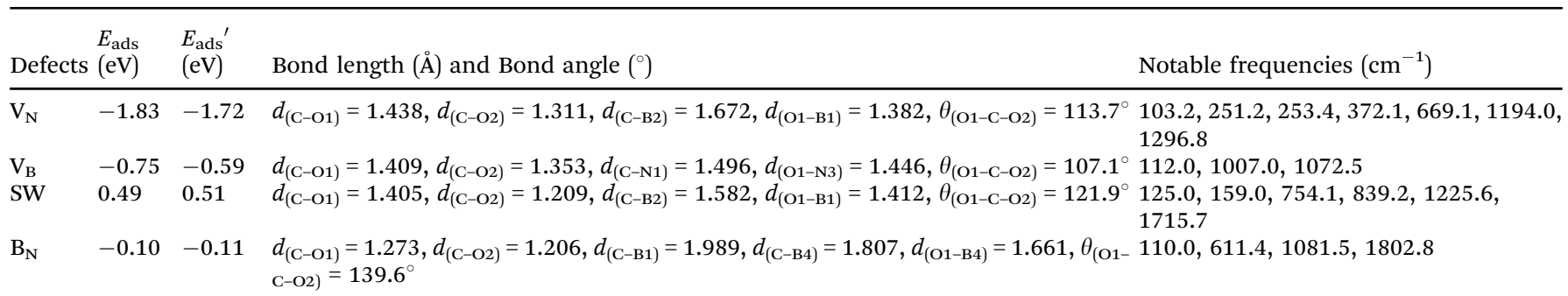




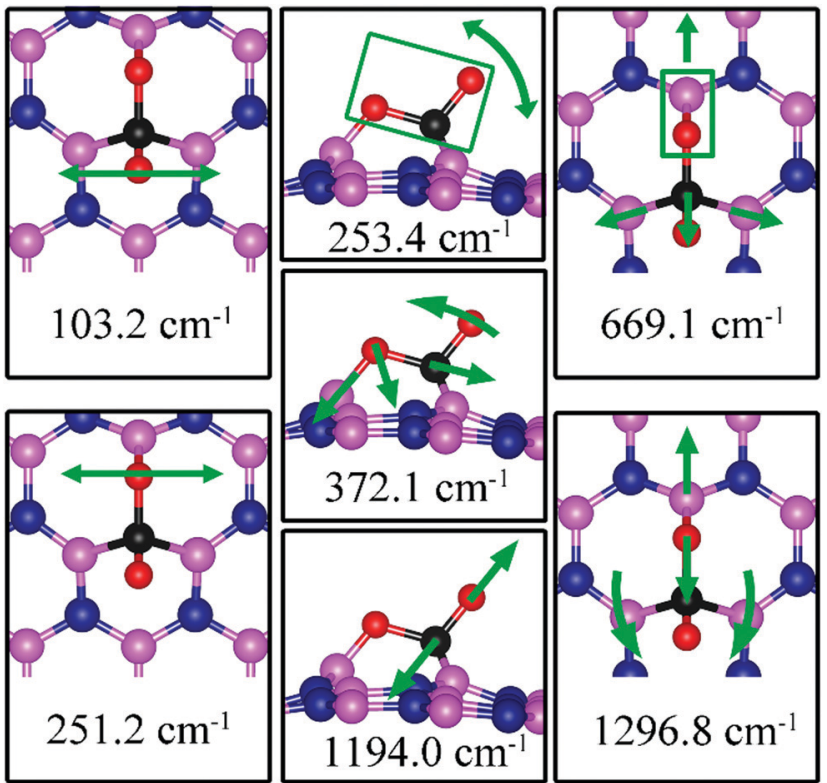

Fig. 15 Notable vibrational modes of $\mathrm{CO}_{2}$ adsorption on $d h-\mathrm{BN}$ with $\mathrm{V}_{\mathrm{N}}$ The magenta, blue, black and red balls represent the $\mathrm{B}, \mathrm{N}, \mathrm{C}$, and $\mathrm{O}$ atoms, respectively. Arrows indicate vibrational patterns. The rectangular encloses the atoms that move together

electron density (violet region) between the $\mathrm{C}$ and $\mathrm{O}$ atoms, consistent with the weakening of $\mathrm{C}-\mathrm{O}$ bonds. PDOS shown in Fig. 14b indicates hybridizations of electronic states of the adsorbed $\mathrm{CO}_{2}$ molecule and defects states of $d h$-BN with $\mathrm{V}_{\mathrm{B}}$, confirming the chemisorption of the molecule. It also suggests that the dangling bonds at the $\mathrm{N}$ atoms at the defect site of $d h$-BN are partially saturated, as the contributions of $\mathrm{N}$ atoms to the defect states are reduced as compared to that in the absence of $\mathrm{CO}_{2}$.

Similar to the case of $\mathrm{CO}_{2}$ adsorbed on the $d h$-BN with $\mathrm{V}_{\mathrm{N}}$, the molecule is found to adsorb with a bent configuration on $\mathrm{V}_{\mathrm{B}}$. Thus the normal vibrational modes of $\mathrm{CO}_{2}$ molecule (symmetric stretching, antisymmetric stretching, bending) are not present. We found again three notable vibrational modes that show contributions from the adsorbed $\mathrm{CO}_{2}$ molecules, as shown in Fig. 16, with their frequencies listed in Table 3. The CO stretching mode that points outward from $d h$-BN has a frequency of $1007.0 \mathrm{~cm}^{-1}$. This mode is also infrared active and we assign this mode as a signature for the identification of the adsorption of $\mathrm{CO}_{2}$ on the $d h$-BN with $\mathrm{V}_{\mathrm{N}}$.

\section{$\mathrm{CO}_{2}$ adsorption on SW}

On $d h$-BN with $\mathrm{SW}, \mathrm{CO}_{2}$ binds at two B sites with an adsorption energy of $+0.49 \mathrm{eV}$ ( $0.51 \mathrm{eV}$ with $\mathrm{ZPE}$ correction) indicating that the adsorption is endothermic. As shown in Fig. 13c, the adsorbed $\mathrm{CO}_{2}$ pulls the $\mathrm{B}$ atom out of the $d h$-BN plane. The two $\mathrm{C}-\mathrm{O}$ bond lengths are found to be $1.405 \AA$ (C-O1) and $1.209 \AA$ (C-O2). The bond lengths, $d_{(\mathrm{C}-\mathrm{B} 2)}$ and $d_{(\mathrm{O} 1-\mathrm{B} 1)}$, are 1.582 and $1.412 \AA$, respectively. Bader charge analysis shows 1.467 electrons transferred from $d h$-BN with SW to the molecule. CDD plot of $\mathrm{CO}_{2}$ on $d h$-BN with SW, as shown in Fig. 13c, indicates a sizable electron density accumulation (green region) between
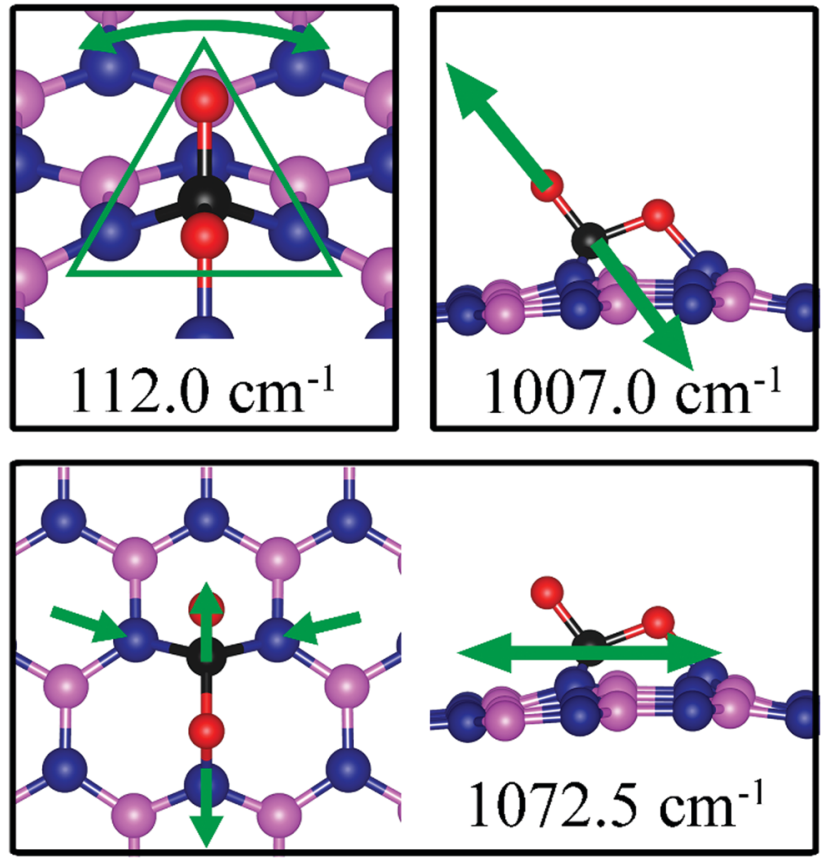

Fig. 16 Notable vibrational modes of $\mathrm{CO}_{2}$ adsorption on $d h-\mathrm{BN}$ with $\mathrm{V}_{\mathrm{B}}$. The magenta, blue, black and red balls represent the $\mathrm{B}, \mathrm{N}, \mathrm{C}$, and $\mathrm{O}$ atoms, respectively. Arrows indicate vibrational patterns. The trapezium-like shape in $141.8 \mathrm{~cm}^{-1}$ mode encloses the group of atoms that concertedly vibrate together.

the $\mathrm{B}$ and $\mathrm{C}$ atoms and between the $\mathrm{B}$ and $\mathrm{O}$ atoms, suggesting covalent bond formation between the pairs of atoms and a large loss of electron density in the region between the B1 and B2 atoms, indicating weakening of the B-B bond of the defect. PDOS, as shown in Fig. 14c, indicates that the conduction band moves toward lower energy while the valence band shifts toward higher energy, as in the case of $\mathrm{CO}$ on $d h$-BN with SW. It also shows that contributions from B1, B2, N1, and N2 atoms to the defect states are still dominant while only a small contribution of the adsorbed $\mathrm{CO}_{2}$ is observed. As in the case of $\mathrm{CO}$ adsorption on SW, we find a preferred physisorbed state of $\mathrm{CO}_{2}$ with a binding energy of $-0.14 \mathrm{eV}$. By performing Nudged Elastic Band calculation (see Fig. S1 in ESI $\dagger$ ), we find a barrier of $1.71 \mathrm{eV}$ between the physisorbed and chemisorbed configurations. However, if $\mathrm{CO}_{2}$ is already chemically adsorbed on SW, the reverse barrier is also very high at $1.08 \mathrm{eV}$ (see Fig. $\mathrm{S} 1$ in $\mathrm{ESI} \dagger$ ), which means $\mathrm{CO}_{2}$ will remain chemically adsorbed. Interestingly, the above findings lead to the conclusion that $\mathrm{CO}_{2}$ chemisorption on SW is not weak.

The calculated vibrational frequencies for the above configurations display four notable modes that are localized at or near the adsorbed $\mathrm{CO}_{2}$. Their vibrational patterns are shown in Fig. 17 and their frequencies are listed in Table 3. We find the CO stretching mode that points outward from the defect to have a frequency of $1715.7 \mathrm{~cm}^{-1}$. This mode is infrared active and could be the signature of this defect site.

\section{$\mathrm{CO}_{2}$ adsorption on $\mathrm{B}_{\mathrm{N}}$}

On $d h-\mathrm{BN}$ with $\mathrm{B}_{\mathrm{N}}$ as shown in Fig. 13d, the adsorbed $\mathrm{CO}_{2}$ molecule undergoes structural distortion to a bent geometry 


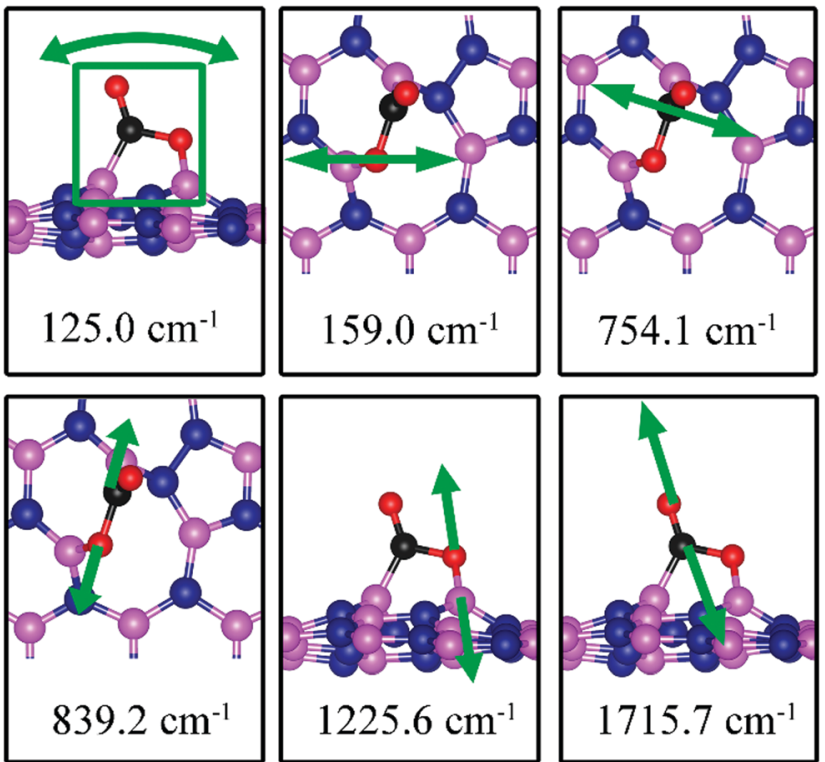

Fig. 17 Notable vibrational modes of $\mathrm{CO}_{2}$ adsorption on $d h-\mathrm{BN}$ with SW. The magenta, blue, black and red balls represent the $\mathrm{B}, \mathrm{N}, \mathrm{C}$, and $\mathrm{O}$ atoms, respectively. Arrows indicate vibrational patterns. The square in $124.6 \mathrm{~cm}^{-1}$ mode figure (left) encloses all atoms that concertedly vibrate together.

with $\mathrm{C}-\mathrm{O}$ bond order reduction similar to that reported for $\mathrm{CO}_{2}$ chemisorbed on B-rich BNNTs. ${ }^{42}$ The $\mathrm{O} 1-\mathrm{C}-\mathrm{O} 2$ bond angle is $139.6^{\circ}$, and the $\mathrm{C}-\mathrm{O} 1$ and $\mathrm{C}-\mathrm{O} 2$ bonds are significantly elongated to 1.273 and $1.206 \AA$, respectively, indicating bond-order reductions. The $\mathrm{B} 4$ atom at the defect site is pulled out of $d h-\mathrm{BN}$ plane considerably by $\sim 1 \AA$. The adsorption energy of $\mathrm{CO}_{2}$ adsorbed at the $\mathrm{B}_{\mathrm{N}}$ is found to be $-0.10 \mathrm{eV}(-0.11 \mathrm{eV}$ with $\mathrm{ZPE}$ correction), which is significantly lower than that of $-0.74 \mathrm{eV}$ reported for B-rich BNNTs. ${ }^{42}$ Bader charge analysis shows 0.710 electrons are transferred from the $d h$-BN to the molecule. CDD plot of $\mathrm{CO}_{2}$ on $d h$-BN with $\mathrm{B}_{\mathrm{N}}$, as shown in Fig. 13d, indicates a sizable electron density accumulation (green region) in the region between the $\mathrm{B}$ and $\mathrm{C}$ atoms and between the $\mathrm{B}$ and $\mathrm{O}$ atoms and a large loss of electron density (violet region) in the region between the $\mathrm{B} 1$ and $\mathrm{B} 2$ atoms, indicating the weakening of $\mathrm{B}-\mathrm{B}$ bond. The $\mathrm{CO}_{2}$ adsorption on $d h$-BN with $\mathrm{B}_{\mathrm{N}}$ thus causes the density of states to shift toward higher energy (Fig. 14d), consistent with the direction of electron transfer from $d h$-BN to the $\mathrm{CO}_{2}$ molecule.

We found two notable vibrational modes that display a large contribution from the adsorbed $\mathrm{CO}_{2}$ molecule. The displacement patterns of these modes are shown in Fig. 18 and their frequencies are listed in Table 3 . The $\mathrm{CO}$ stretching mode that points outward from the $d h$-BN plane has a frequency of $1802.8 \mathrm{~cm}^{-1}$. This mode is infrared active and we assign it as the signature of the adsorption configuration discussed above.

\section{Propensity for chemical activity of $\boldsymbol{d} \boldsymbol{h}$-BN}

In this section we extrapolate the implications of our findings of adsorption energies of the molecules toward catalytic application of $d h-\mathrm{BN}$ for hydrogenation of $\mathrm{CO}$ and $\mathrm{CO}_{2}$, bearing in mind that to facilitate these reactions we should be taking into
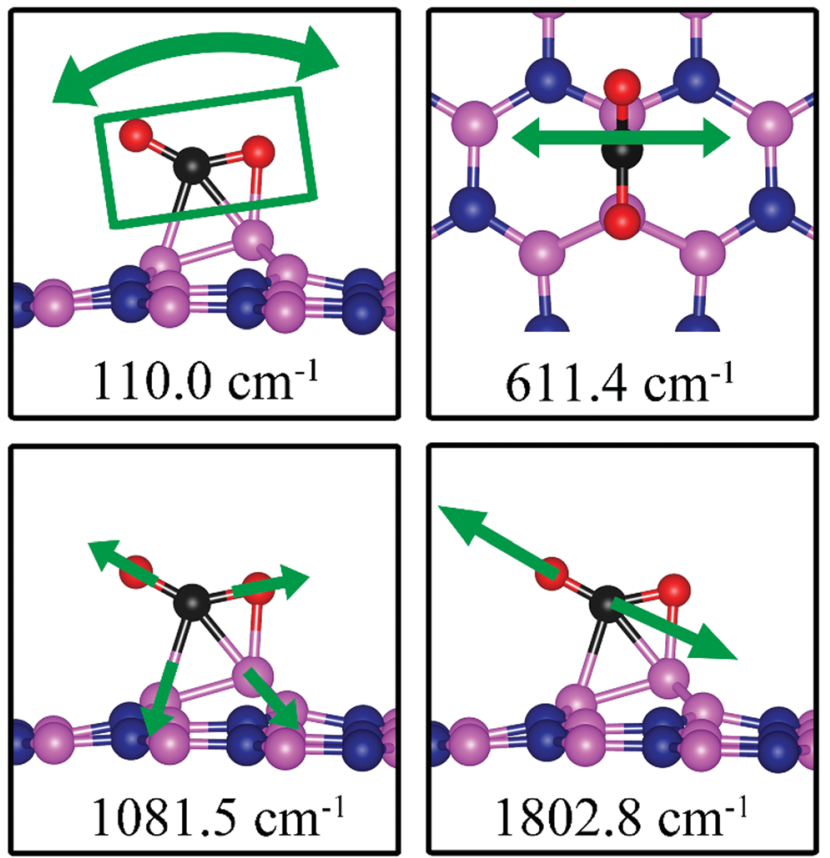

Fig. 18 Notable vibrational modes of $\mathrm{CO}_{2}$ adsorption on $d h-\mathrm{BN}$ with $\mathrm{B}_{\mathrm{N}}$. The magenta, blue, black and red balls represent the B, N, C, and $\mathrm{O}$ atoms, respectively. Arrows indicate vibrational patterns. The rectangular in the left figure encloses atoms that concertedly vibrate together.

account co-adsorption and diffusion of syngas molecules, and subsequent reaction steps which are beyond the scope of the present study. Fig. 19 shows comparison of the adsorption energy of $2 \mathrm{H}, \mathrm{CO}$, and $\mathrm{CO}_{2}$. We do not include $\mathrm{H}_{2}$ in the above since it only physisorbs on these $d h$-BN. As indicated by the positive energies in Fig. 19, on $d h$-BN with SW, the adsorption of $2 \mathrm{H}, \mathrm{CO}$ and $\mathrm{CO}_{2}$ are endothermic, suggesting low chemical reactivity of $d h$-BN with $\mathrm{SW}$. This is consistent with the fact that $\mathrm{B}$ and $\mathrm{N}$ atoms have 3 valence electrons and in SW defect each has 3 covalent bonds. Creating covalent bonds with adsorbate molecules is thus energetically not favorable. In the case of the adsorption of $2 \mathrm{H}, \mathrm{B}-\mathrm{H}$ and $\mathrm{N}-\mathrm{H}$ bond formations lead to the breaking of the original B-N bond of the SW defect. With $\mathrm{CO}_{2}$ adsorption $\mathrm{B}-\mathrm{C}$ and $\mathrm{B}-\mathrm{O}$ bond formations lead to breaking of the original $\mathrm{B}-\mathrm{B}$ bond of the $\mathrm{SW}$ defect to compensate the formations of $\mathrm{B}-\mathrm{C}$ and $\mathrm{B}-\mathrm{O}$ bonds, i.e., to warrant that each $\mathrm{B}$ atom has 3 covalent bonds. The energy gained in the formations of new bonds and energy lost in the breaking of the original bond in SW defect compete with each other. Their cooperative effect does not offer an exothermic process. Because of such unfavorable adsorptions, hydrogenation of $\mathrm{CO}$ or $\mathrm{CO}_{2}$ are not feasible on such a defect.

On $d h$-BN with $\mathrm{V}_{\mathrm{B}}$, on the other hand, adsorption energies of $2 \mathrm{H}$ and $\mathrm{CO}$ are similar and in the range from -4 to $-5 \mathrm{eV}$, suggesting rather strong adsorption. Such strong adsorption, however, often leads to difficulties, i.e., high energetics barrier, in the removal or diffusion of the adsorbates (reactants) and for co-adsorption to occur. It is most likely that the $\mathrm{V}_{\mathrm{B}}$ sites will remain occupied by $\mathrm{H}$ or $\mathrm{CO}$ during a reaction, thus deactivating the site. Moreover, with the $\mathrm{V}_{\mathrm{B}}$ defect adsorption of $\mathrm{CO}_{2}$ is 


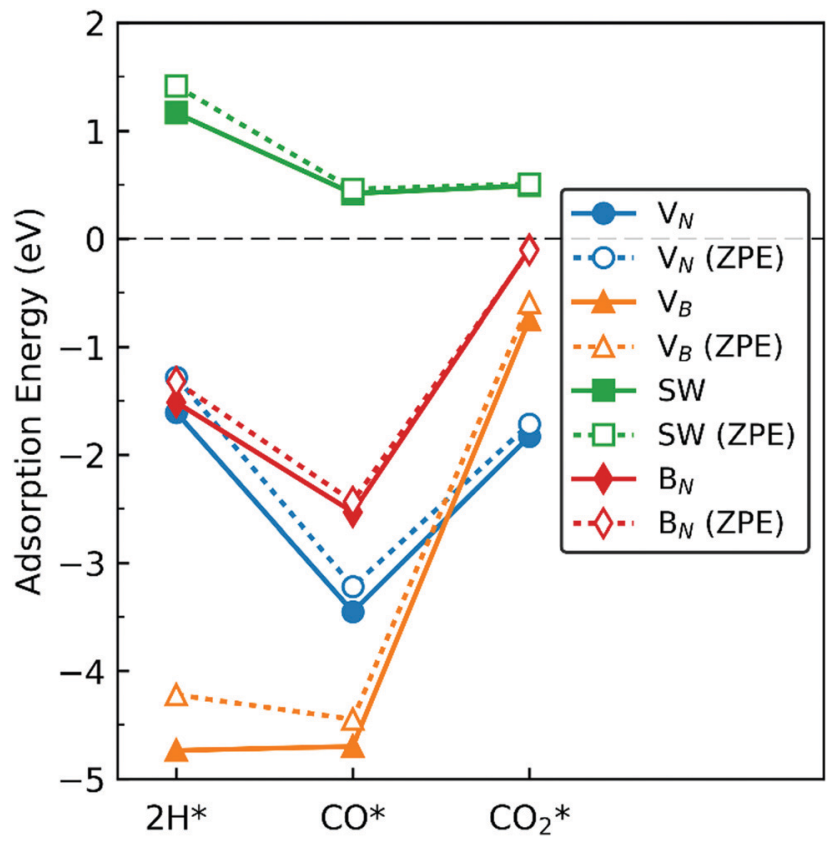

Fig. 19 Adsorption energy of $2 \mathrm{H}, \mathrm{CO}$, and $\mathrm{CO}_{2}$ on the dh-BN with (a) $\mathrm{V}_{\mathrm{N}}$, (b) $V_{B}$, (c) SW, and (d) $B_{N}$, shown with circle, triangle, square, and diamond markers, respectively. Values without and with ZPE corrections are shown in solid and empty markers, respectively. The markers for each defect types are connected with solid or dotted lines for guiding the eyes.

substantially weaker than that of $2 \mathrm{H}$, making it less likely that the site is occupied by $2 \mathrm{H}$. We thus conclude that $d h$ - $\mathrm{BN}$ with $\mathrm{V}_{\mathrm{B}}$ is also not suitable for $\mathrm{CO}_{2}$ hydrogenation. Similarly, on $d h$-BN with $\mathrm{B}_{\mathrm{N}}$, adsorption of $\mathrm{CO}_{2}$ is a weak chemisorption, implying that $\mathrm{CO}_{2}$ could easily desorb before it would have the opportunity to react with other species under reaction conditions. On the other hand, the strength of $2 \mathrm{H}$ and $\mathrm{CO}$ adsorptions are similar and strong for stable adsorption. However, the PDOS for $2 \mathrm{H}$ and $\mathrm{CO}$ on $d h$-BN with $\mathrm{B}_{\mathrm{N}}$, as shown in Fig. 5 and 8, respectively, do not show any mid-gap state, indicating there is no dangling bond available for more molecules to adsorb. The above lead us to surmise that for such a defect co-adsorption is less likely to occur. The situation is different for $d h$ - $\mathrm{BN}$ with $\mathrm{V}_{\mathrm{N}}$. With this particular defect we find that while the binding of CO is strong (adsorption energy of $-3.22 \mathrm{eV}$ with ZPE correction)-suggesting that CO could occupy and deactivate the site-the adsorption energies of $2 \mathrm{H}$ and $\mathrm{CO}_{2}$ are in a similar range that is not too strong to block active sites nor too weak to be unstable. The above comparisons lead us to suggest that $d h$-BN with $\mathrm{V}_{\mathrm{N}}$ could be a potential catalyst for $\mathrm{CO}_{2}$ hydrogenation. This is in agreement with a similar conclusion obtained for the hydrogenation of olefins. ${ }^{6}$

Our results presented above suggest that the strength of binding of the adsorbates depends on the availability of undercoordinated sites of the defects. For the creation of $V_{B}$ and $V_{N}$ vacancy defects, three covalent bonds together with three valence electrons were removed. As a result, the number of electrons in the system is odd and the system is spin-polarized because there is an unpaired electron. More importantly, they create 3 undercoordinated sites at the defects. The situation is different for the case of $\mathrm{BN}$ and SW defects, in which all atoms do have 3 covalent bonds and all electrons are paired. As a result, there is no undercoordinated site at the defect.

\section{Conclusions}

Our systematic analysis of the electronic structure and vibrational dynamics of syngas molecules adsorbed on defect-laden single-layer $h$-BN, using DFT-based calculations, has allowed us to make a distinction among four types of defects $\left(V_{N}, V_{B}, S W\right.$, and $\mathrm{B}_{\mathrm{N}}$ ) that we considered. We find that $\mathrm{CO}$ and $\mathrm{CO}_{2}$ molecules chemisorb on $d h$-BN for each of the four defect types, while $\mathrm{H}_{2}$ only physisorbs on these defects. On the other hand, atomic hydrogen adsorbs on the defects. The above conclusions are also supported by an analysis of the partial density of electronic states and charge density difference plots. In addition, our calculations of vibrational frequencies for the considered adsorptions point to a number of notable vibrational modes that could help in the identification of defect type, through their experimental examination such as infrared absorption spectroscopy of the adsorbed molecules on $d h$-BN.

Our results provide fundamental insights into the interaction of syngas molecules and $d h$-BN through which we can prescreen the defect type that would make the basal plane of the dh-BN catalytically active for $\mathrm{CO}$ and $\mathrm{CO}_{2}$ hydrogenation reactions. In particular, by comparing the adsorption energy of the molecules on $d h$-BN with each of the four defect types, accompanied with analysis of their electronic and geometric structures, we suggest that $d h$ - $\mathrm{BN}$ with $\mathrm{V}_{\mathrm{N}}$ (nitrogen vacancy) as a good candidate catalyst for $\mathrm{CO}_{2}$ hydrogenation. We await experimental results that validate our findings.

On a more general note, although we have focused mostly on the chemical activity of defect-laden $h$-BN in this work, as we mention in the introduction, this interesting low-dimensional material is also being proposed as a single photon emitter in which defects play a major and their characterization is a challenge. Our work provides signatures in the vibrational modes of the adsorbed molecules that are strikingly different for the four defect types and thus could serve to identify them in experiments such as infra-red absorption spectroscopy which is commonly available. We hope our work encourages colleagues to undertake such measurements as a probe of the nature of the defect in $h$-BN that gives rise to several novel properties.

\section{Conflicts of interest}

There are no conflicts to declare.

\section{Acknowledgements}

It is a pleasure to submit this work to a volume dedicated to Peter Toennies with whom TSR has had many fruitful scientific interactions over the years. She also remembers very fondly discussions with JPT at Schloss Ringberg and a particular 
challenging hike in the area which fortunately ended well. We also thank Richard Blair and Hannah Stern for many helpful discussions. This work was supported in part by the U.S. Department of Energy, under Grant DE-FG02-07ER15842. The DFT calculations were performed in part using the computing resources at STOKES in the Advanced Research Computing Center at University of Central Florida, and at the National Energy Research Scientific Computing Center (NERSC). TSR is also indebted to the Miller Foundation, University of California, Berkeley, for the award of a Visiting Miller Professorship which facilitated some of the work presented here.

\section{Notes and references}

1 Y. Lin and J. W. Connell, Nanoscale, 2012, 4, 6908-6939.

2 K. Watanabe, T. Taniguchi and H. Kanda, Nat. Mater., 2004, 3, 404-409.

3 R. Decker, Y. Wang, V. W. Brar, W. Regan, H.-Z. Tsai, Q. Wu, W. Gannett, A. Zettl and M. F. Crommie, Nano Lett., 2011, 11, 2291-2295.

4 Y. Li, Y. Rao, K. F. Mak, Y. You, S. Wang, C. R. Dean and T. F. Heinz, Nano Lett., 2013, 13, 3329-3333.

5 J. Di, J. Xia, M. Ji, B. Wang, S. Yin, Q. Zhang, Z. Chen and H. Li, Appl. Catal., B, 2016, 183, 254-262.

6 D. J. Nash, D. T. Restrepo, N. S. Parra, K. E. Giesler, R. A. Penabade, M. Aminpour, D. Le, Z. Li, O. K. Farha, J. K. Harper, T. S. Rahman and R. G. Blair, ACS Omega, 2016, 1, 1343-1354.

7 J. T. Grant, C. A. Carrero, F. Goeltl, J. Venegas, P. Mueller, S. P. Burt, S. E. Specht, W. P. McDermott, A. Chieregato and I. Hermans, Science, 2016, 354, 1570-1573.

8 T. T. Tran, K. Bray, M. J. Ford, M. Toth and I. Aharonovich, Nat. Nanotechnol., 2016, 11, 37-41.

9 J. Feng, H. Deschout, S. Caneva, S. Hofmann, I. Lončarić, P. Lazić and A. Radenovic, Nano Lett., 2018, 18, 1739-1744.

10 R. Bourrellier, S. Meuret, A. Tararan, O. Stéphan, M. Kociak, L. H. G. Tizei and A. Zobelli, Nano Lett., 2016, 16, 4317-4321.

11 M. Topsakal, E. Aktürk and S. Ciraci, Phys. Rev. B: Condens. Matter Mater. Phys., 2009, 79, 115442.

12 D. Le, T. B. Rawal and T. S. Rahman, J. Phys. Chem. C, 2014, 118, 5346-5351.

13 T. B. Rawal, D. Le and T. S. Rahman, J. Phys.: Condens. Matter, 2017, 29, 415201.

14 T. B. Rawal, D. Le and T. S. Rahman, J. Phys. Chem. C, 2017, 121, 7282-7293.

15 P. E. Evans, H. K. Jeong, Z. Hooshmand, D. Le, T. B. Rawal, S. N. Alvillar, L. Bartels, T. S. Rahman and P. A. Dowben, J. Phys. Chem. C, 2018, 122, 10042-10049.

16 C. S. Merida, D. Le, E. M. Echeverria, A. E. Nguyen, T. B. Rawal, S. Naghibi Alvillar, V. Kandyba, A. Al-Mahboob, Y. Losovyj, K. Katsiev, M. D. Valentin, C.-Y. Huang, M. J. Gomez, I. H. Lu, A. Guan, A. Barinov, T. S. Rahman,
P. A. Dowben and L. Bartels, J. Phys. Chem. C, 2018, 122, 267-273.

17 H. Li, C. Tsai, A. L. Koh, L. Cai, A. W. Contryman, A. H. Fragapane, J. Zhao, H. S. Han, H. C. Manoharan, F. Abild-Pedersen, J. K. Norskov and X. Zheng, Nat. Mater., 2016, 15, 48-53.

18 B. Huang and H. Lee, Phys. Rev. B: Condens. Matter Mater. Phys., 2012, 86, 245406.

19 C. Jin, F. Lin, K. Suenaga and S. Iijima, Phys. Rev. Lett., 2009, 102, 195505.

20 R. Schimmenti, R. Cortese, D. Duca and M. Mavrikakis, ChemCatChem, 2017, 9, 1610-1620.

21 S. Lin, X. Ye, R. S. Johnson and H. Guo, J. Phys. Chem. C, 2013, 117, 17319-17326.

22 X. Liu, T. Duan, C. Meng and Y. Han, RSC Adv., 2015, 5, 10452-10459.

23 Q. Sun, Z. Li, D. J. Searles, Y. Chen, G. M. Lu and A. Du, J. Am. Chem. Soc., 2013, 135, 8246-8253.

24 A. L. Gibb, N. Alem, J. H. Chen, K. J. Erickson, J. Ciston, A. Gautam, M. Linck and A. Zettl, J. Am. Chem. Soc., 2013, 135, 6758-6761.

25 C. K. Oliveira, E. F. A. Gomes, M. C. Prado, T. V. Alencar, R. Nascimento, L. M. Malard, R. J. C. Batista, A. B. de Oliveira, H. Chacham, A. M. de Paula and B. R. A. Neves, Nano Res., 2015, 8, 1680-1688.

26 I. N. Remediakis, F. Abild-Pedersen and J. K. Norskov, J. Phys. Chem. B, 2004, 108, 14535-14540.

27 Y. Choi and P. Liu, J. Am. Chem. Soc., 2009, 131, 13054-13061.

28 Y. Yang, J. Evans, J. A. Rodriguez, M. G. White and P. Liu, Phys. Chem. Chem. Phys., 2010, 12, 9909-9917.

29 H. Wu, X. Fan and J.-L. Kuo, Int. J. Hydrogen Energy, 2012, 37, 14336-14342.

30 W. Wang, S. Wang, X. Ma and J. Gong, Chem. Soc. Rev., 2011, 40, 3703-3727.

31 P. Giannozzi, S. Baroni, N. Bonini, M. Calandra, R. Car, C. Cavazzoni, D. Ceresoli, G. L. Chiarotti, M. Cococcioni, I. Dabo, A. Dal Corso, S. de Gironcoli, S. Fabris, G. Fratesi, R. Gebauer, U. Gerstmann, C. Gougoussis, A. Kokalj, M. Lazzeri, L. Martin-Samos, N. Marzari, F. Mauri, R. Mazzarello, S. Paolini, A. Pasquarello, L. Paulatto, C. Sbraccia, S. Scandolo, G. Sclauzero, A. P. Seitsonen, A. Smogunov, P. Umari and R. M. Wentzcovitch, J. Phys.: Condens. Matter, 2009, 21, 395502.

32 P. E. Blöchl, Phys. Rev. B: Condens. Matter Mater. Phys., 1994, 50, 17953-17979.

33 G. Kresse and D. Joubert, Phys. Rev. B: Condens. Matter Mater. Phys., 1999, 59, 1758-1775.

34 K. Lee, E. D. Murray, L. Z. Kong, B. I. Lundqvist and D. C. Langreth, Phys. Rev. B: Condens. Matter Mater. Phys., 2010, 82, 081101.

35 W. Tang, E. Sanville and G. Henkelman, J. Phys.: Condens. Matter, 2009, 21, 084204.

36 R. F. Bader, Acc. Chem. Res., 1985, 18, 9-15.

37 A. Togo and I. Tanaka, Scr. Mater., 2015, 108, 1-5. 
38 S. Azevedo, J. R. Kaschny, C. M. C. de Castilho and F. de Brito Mota, Eur. Phys. J. B, 2009, 67, 507-512.

39 S. Yu, L. Li, Z. Lai, J. Hao and K. Zhang, Mater. Res. Express, 2017, 4, 116302.

40 R. Muhammad, Y. Shuai and H.-P. Tan, J. Mater. Chem. C, 2017, 5, 8112-8127.

41 R. Wang, J. Yang, X. Wu and S. Wang, Nanoscale, 2016, 8, 8210-8219.

42 H. Choi, Y. C. Park, Y. H. Kim and Y. S. Lee, J. Am. Chem. Soc., 2011, 133, 2084-2087.

43 Z. L. Liu, Q. Z. Xue, T. Zhang, Y. H. Tao, C. C. Ling and M. X. Shan, J. Phys. Chem. C, 2013, 117, 9332-9339.
44 K. Mao, L. Li, W. Zhang, Y. Pei, X. C. Zeng, X. Wu and J. Yang, Sci. Rep., 2014, 4, 5441.

45 Y.-H. Zhang, K.-G. Zhou, X.-C. Gou, K.-F. Xie, H.-L. Zhang and Y. Peng, Chem. Phys. Lett., 2010, 484, 266-270.

46 C. Tabtimsai, A. Nonsri, N. Gratoo, N. Massiri, P. Suvanvapee and B. Wanno, Monatsh. Chem., 2014, 145, 725-735.

47 G. Henkelman and H. Jonsson, J. Chem. Phys., 2000, 113, 9978-9985.

48 G. Henkelman, B. P. Uberuaga and H. Jonsson, J. Chem. Phys., 2000, 113, 9901-9904.

49 Y. Jiao, A. Du, Z. Zhu, V. Rudolph, G. Q. Lu and S. C. Smith, Catal. Today, 2011, 175, 271-275. 